\title{
On the Fučík spectrum of the scalar $p$-Laplacian with indefinite integrable weights
}

\author{
Wei Chen ${ }^{1}$, Jifeng Chu', Ping Yan ${ }^{2 *}$ and Meirong Zhang ${ }^{2}$
}

\section{"Correspondence:}

pyan@math.tsinghua.edu.cn

${ }^{2}$ Department of Mathematical

Sciences, Tsinghua University,

Beijing, 100084, People's Republic of China

Full list of author information is available at the end of the article

\begin{abstract}
In this paper, we study the structure of the Fučík spectrum $\Pi_{p}^{D / N}(a, b)$ of Dirichlet and Neumann problems for the scalar $p$-Laplacian with indefinite weights $a, b \in L^{1}[0,1]$. Besides the trivial horizontal lines and vertical lines, it will be shown that, confined to each quadrant of $\mathbb{R}^{2}, \Pi_{p}^{D / N}(a, b)$ is made up of zero, an odd number of, or a double sequence of hyperbolic like curves. These hyperbolic like curves are continuous and strictly monotonic, and they have horizontal and vertical asymptotic lines. The number of the hyperbolic like curves is determined by the Dirichlet and Neumann half-eigenvalues of the $p$-Laplacian with weights $a$ and $b$. The asymptotic lines will be estimated by using Sturm-Liouville eigenvalues of the $p$-Laplacian with a weight $a$ orb.
\end{abstract}

MSC: 34B09; 34B15; 34L05

Keywords: indefinite weights; $p$-Laplacian; Fučík spectrum; spectral structure

\section{Introduction}

Fučík spectrum was first introduced for the Laplacian on a bounded domain $\Omega \subset \mathbb{R}^{N}, N \geq$ 1, by Dancer [1] and by Fučík [2] in the 1970s, in connection with the study of semilinear elliptic boundary value problems with jumping nonlinearities. Thereafter this important concept was generalized to the $p$-Laplacian, $p>1$. See [3] and references therein.

In this paper, we are concerned with the Fućík spectrum of the scalar $p$-Laplacian

$$
\phi_{p}(x)= \begin{cases}|x|^{p-2} x, & x \neq 0 \\ 0, & x=0\end{cases}
$$

Given $a, b \in L^{1}[0,1]$, taking the notations $x_{ \pm}=\max \{ \pm x, 0\}$, let us consider the ODE

$$
\left(\phi_{p}\left(x^{\prime}\right)\right)^{\prime}+(\lambda+a(t)) \phi_{p}\left(x_{+}\right)-(\mu+b(t)) \phi_{p}\left(x_{-}\right)=0, \quad \text { a.e. } t \in[0,1],
$$

in which $a, b$ are called potentials, and the ODE

$$
\left(\phi_{p}\left(x^{\prime}\right)\right)^{\prime}+\lambda a(t) \phi_{p}\left(x_{+}\right)-\mu b(t) \phi_{p}\left(x_{-}\right)=0, \quad \text { a.e. } t \in[0,1],
$$

in which $a, b$ are called weights. For a pair of potentials $a$ and $b$, the Fučík spectra $\Sigma_{p}^{D}(a, b)$ and $\Sigma_{p}^{N}(a, b)$ are defined as the sets of those $(\lambda, \mu) \in \mathbb{R}^{2}$ such that equation (1.1) has non-

@2014 Chen et al.; licensee Springer. This is an Open Access article distributed under the terms of the Creative Commons Attribution License (http://creativecommons.org/licenses/by/2.0), which permits unrestricted use, distribution, and reproduction in any medium, provided the original work is properly cited. 
trivial solutions satisfying the Dirichlet boundary condition

$$
x(0)=x(1)=0
$$

and the Neumann boundary condition

$$
x^{\prime}(0)=x^{\prime}(1)=0
$$

respectively. Similarly, for a pair of weights $a$ and $b$, the Fućík spectra $\Pi_{p}^{D}(a, b)$ and $\Pi_{p}^{N}(a, b)$ are defined as the sets of those $(\lambda, \mu) \in \mathbb{R}^{2}$ such that equation (1.2) has non-trivial solutions satisfying the corresponding boundary conditions (1.3) and (1.4), respectively.

The Fučík spectra $\Sigma_{p}^{D}(a, b)$ and $\Sigma_{p}^{N}(a, b)$ have been comprehensively understood in [4]: each of them is composed of one horizontal line, one vertical line and a double sequence of differentiable, strictly decreasing, hyperbolic like curves; asymptotic lines of these hyperbolic like curves are given by using (Sturm-Liouville) eigenvalues of the $p$-Laplacian with a potential; moreover, these curves have a strong continuous dependence on the potentials.

Compared with potentials, indefinite weights will add difficulties to the study of the Fučík spectra. Alif [5] studied $\Pi_{p}^{D}(a, b)$ and $\Pi_{p}^{N}(a, b)$ by means of 'zero functions', where the weights $a$ and $b$ were assumed to be sign-changing (i.e., $a_{ \pm} \not \equiv 0$ and $\left.b_{ \pm} \not \equiv 0\right)$ continuous functions without 'singular points' (which is a technical hypothesis). Their main results are as follows. Besides the trivial horizontal lines and vertical lines, confined to each quadrant of $\mathbb{R}^{2}, \Pi_{p}^{D / N}(a, b)$ consists of an odd number of or infinitely many hyperbolic like curves. The asymptotic behavior of the first non-trivial curves in each quadrant was also studied. It was observed that for instance the first curve of $\Pi_{p}^{N}(a, b)$ in $\mathbb{R}^{+} \times \mathbb{R}^{+}$is not asymptotic on any side to the trivial horizontal and vertical lines. In other words, there are always gaps between its asymptotic lines and the trivial horizontal and vertical lines. However, the exact asymptotic lines were not found in that paper.

In this paper, we are interested in $\Pi_{p}^{D}(a, b)$ and $\Pi_{p}^{N}(a, b)$, where the weights $a, b \in L^{1}[0,1]$ are assumed to be indefinite (i.e., $a$ and $b$ may or may not change sign). In this case, since the weights are integrable, the method employed in [5] does not work anymore. Using the Prüfer transformation, we convert the second-order ODE (1.2) into a system of firstorder ODEs (3.2) and (3.3), for the argument $\theta$ and the radius $r$, respectively. The ODE (3.2) for $\theta$ turns to be independent of $r$, and the boundary conditions (1.3) and (1.4) can be characterized by the solutions of equation (3.2), therefore the Fučík spectra $\Pi_{p}^{D}(a, b)$ and $\Pi_{p}^{N}(a, b)$ are completely determined by this first-order ODE (3.2). The solutions of equation (3.2) admit (strong) continuity and Fréchet differentiability in the weights. Based on these properties, we will finally reveal the structure of the Fučík spectra. Our main results are as follows.

(i) Besides at most two vertical lines and two horizontal lines, $\Pi_{p}^{D / N}(a, b)$ confined to each quadrant of $\mathbb{R}^{2}$ is made up of zero, an odd number of, or a double sequence of continuous, strictly monotonic, hyperbolic like curves.

(ii) The number of those trivial lines in $\Pi_{p}^{D / N}(a, b)$ is determined by the Dirichlet and Neumann eigenvalues of the $p$-Laplacian.

(iii) The number of the hyperbolic like curves in $\Pi_{p}^{D / N}(a, b)$ is determined by the Dirichlet and Neumann half-eigenvalues of the $p$-Laplacian. 
(iv) All the hyperbolic like curves have vertical and horizontal asymptotic lines, and these asymptotic lines will be estimated by using (Sturm-Liouville) eigenvalues of the $p$-Laplacian.

(v) If the weights $a$ and $b$ are positive, the structure of $\Pi_{p}^{D / N}(a, b)$ is comparable with that of $\Sigma_{p}^{D / N}(a, b)$, the case with potentials. More precisely, $\Pi_{p}^{D / N}(a, b)$ is composed of one horizontal line, one vertical line and a double sequence of differentiable, strictly decreasing, hyperbolic like curves in the quadrant $\mathbb{R}^{+} \times \mathbb{R}^{+}$. And all asymptotic lines of these hyperbolic like curves will be given by using (Sturm-Liouville) eigenvalues of the $p$-Laplacian.

The paper is organized as follows. In Section 2, we will give some preliminary results. Section 3 is devoted to $\Pi_{p}^{D}(a, b)$. We first decompose $\Pi_{p}^{D}(a, b)$ in Section 3.1, according to the number of zeroes of the eigenfunctions. Sections 3.2 and 3.3 are devoted to eigenvalues and half-eigenvalues of the $p$-Laplacian, respectively. The results in these two subsections enables us to finally determine the structure of $\Pi_{p}^{D}(a, b)$ in Section 3.4. For a pair of positive weights $a$ and $b$, we can get more information on $\Pi_{p}^{D}(a, b)$ and the results are given in Section 3.5. The Fućík spectrum $\Pi_{p}^{N}(a, b)$ can be studied by similar arguments and we just list the results in Section 4.

\section{Preliminary results}

Given an exponent $p \in(1, \infty)$, denote by $p^{*}$ the conjugate number of $p$, namely $p^{*}=\frac{p}{p-1}$. The initial value problem

$$
\left\{\begin{array}{l}
x^{\prime}(t)=-\phi_{p^{*}}(y), \quad y^{\prime}(t)=\phi_{p}(x) \\
x(0)=1, \quad y(0)=0
\end{array}\right.
$$

has a unique solution $\left(\cos _{p} t, \sin _{p} t\right), t \in \mathbb{R}$. The functions $\cos _{p} t$ and $\sin _{p} t$ are the so-called $p$-cosine and $p$-sine because they possess properties similar to those of the standard cosine and sine, as shown in the following lemma.

Lemma $2.1([6,7])$ The p-cosine and p-sine have the following properties.

(i) Both $\cos _{p}$ t and $\sin _{p}$ t are $2 \pi_{p}$-periodic, where

$$
\pi_{p}=2 \int_{0}^{(p-1)^{1 / p}} \frac{d s}{\left(1-s^{p}(p-1)\right)^{1 / p}}=\frac{2 \pi(p-1)^{1 / p}}{p \sin (\pi / p)}
$$

(ii) $\cos _{p} t$ is even in $t$ and $\sin _{p} t$ is odd in $t$;

(iii) $\cos _{p}\left(t+\pi_{p}\right)=-\cos _{p} t$ and $\sin _{p}\left(t+\pi_{p}\right)=-\sin _{p} t$ for all $t$;

(iv) $\cos _{p} t=0$ if and only if $t=\pi_{p} / 2+n \pi_{p}, n \in \mathbb{Z}$, and $\sin _{p} t=0$ if and only if $t=n \pi_{p}$, $n \in \mathbb{Z}$

(v) $\cos _{p}^{\prime} t=-\phi_{p^{*}}\left(\sin _{p} t\right)$ and $\sin _{p}^{\prime} t=\phi_{p}\left(\cos _{p} t\right)$; and

(vi) $\left|\cos _{p} t\right|^{p}+(p-1)\left|\sin _{p} t\right|^{p^{*}} \equiv 1$.

Remark 2.1 For any $p>1$, one has $\pi_{p}>2$. In fact, if $p=2$, then $\pi_{p}=\pi>2$. If $p>2$, then

$$
\pi_{p}=\frac{2 \pi(p-1)^{1 / p}}{p \sin (\pi / p)}>\frac{2 \pi(p-1)^{1 / p}}{p \cdot(\pi / p)}=2(p-1)^{1 / p}>2
$$


If $1<p<2$, then

$$
\pi_{p}=\frac{2 \pi(p-1)^{1 / p}}{p \sin (\pi / p)}=\frac{2 \pi(p-1)^{1 / p}}{p \sin (\pi-\pi / p)}>\frac{2 \pi(p-1)^{1 / p}}{p \cdot(\pi-\pi / p)}=2(p-1)^{\frac{1}{p}-1}>2 .
$$

Given $a, b \in L^{1}[0,1]$, consider the equation

$$
\left(\phi_{p}\left(x^{\prime}\right)\right)^{\prime}+a(t) \phi_{p}\left(x_{+}\right)-b(t) \phi_{p}\left(x_{-}\right)=0, \quad \text { a.e. } t \in[0,1] .
$$

Let $y=-\phi_{p}\left(x^{\prime}\right)$. Via the $p$-polar coordinates (or Prüfer transformation)

$$
x=r^{2 / p} \cos _{p} \theta, \quad y=r^{2 / p^{*}} \sin _{p} \theta,
$$

we can transform equation (2.1) into the following equations for $r$ and $\theta$ :

$$
\begin{aligned}
& \theta^{\prime}=A(t, \theta ; a, b):=a(t)\left(\cos _{p} \theta\right)_{+}^{p}+b(t)\left(\cos _{p} \theta\right)_{-}^{p}+(p-1)\left|\sin _{p} \theta\right|^{p^{*}}, \\
& (\log r)^{\prime}=\frac{p}{2}\left((a(t)-1)\left(\cos _{p} \theta\right)_{+}^{p-1}+(b(t)-1)\left(\cos _{p} \theta\right)_{-}^{p-1}\right) \phi_{p^{*}}\left(\sin _{p} \theta\right) .
\end{aligned}
$$

Note that equation (2.3) for $\theta$ is independent of $r$. Given $t_{0} \in[0,1]$ and $\theta_{0} \in \mathbb{R}$, denote by $\left(\theta\left(t ; t_{0}, \theta_{0}, a, b\right), r\left(t ; t_{0}, \theta_{0}, a, b\right)\right), t \in[0,1]$, the unique solution of system (2.3)-(2.4) satisfying $\theta\left(t_{0} ; t_{0}, \theta_{0}, a, b\right)=\theta_{0}$ and $r\left(1 ; t_{0}, \theta_{0}, a, b\right)=1$. Let

$$
\Theta\left(\theta_{0}, a, b\right):=\theta\left(1 ; 0, \theta_{0}, a, b\right)
$$

The $p$-polar coordinates (2.2), one can verify that equation (2.1) has a non-trivial solution

$$
X\left(t ; \theta_{0}, a, b\right):=\left(r\left(t ; 0, \theta_{0}, a, b\right)\right)^{2 / p} \cos _{p}\left(\theta\left(t ; 0, \theta_{0}, a, b\right)\right) .
$$

One basic observation on equation (2.3) is that the vector field $A(t, \theta ; a, b)=1>0$ at those $\theta$ such that $\cos _{p} \theta=0$, i.e., $\theta=-\frac{\pi_{p}}{2}+m \pi_{p}, m \in \mathbb{Z}$. Since $a(t)$ and $b(t)$ are only integrable, the derivative $\theta^{\prime}(t)$ at any specific $t$ is meaningless. However, one can still use such an observation to obtain the following property, called quasi-monotonicity. We refer the readers to [7, Lemma 2.3] for a detailed proof.

Lemma 2.2 Given $a, b \in L^{1}[0,1], t_{0} \in[0,1)$ and $\theta_{0} \in \mathbb{R}$, let $\theta(t)=\theta\left(t ; t_{0}, \theta_{0}, a, b\right)$ be the solution of equation (2.3). If $\theta\left(t_{0}\right) \geq-\frac{\pi_{p}}{2}+m \pi_{p}$ for some $m \in \mathbb{Z}$, then

$$
\theta(t)>-\frac{\pi_{p}}{2}+m \pi_{p}, \quad \forall t \in\left(t_{0}, 1\right]
$$

Denote by $w_{1}$ the weak topology in $L^{1}[0,1]$. By $g_{n} \rightarrow g_{0}$ in $\left(L^{1}[0,1], w_{1}\right)$, or $g_{n} \stackrel{w_{1}}{\rightarrow} g_{0}$, we mean that

$$
\int_{0}^{1} g_{n}(t) f(t) d t \rightarrow \int_{0}^{1} g_{0}(t) f(t) d t, \quad \forall f \in L^{\infty}[0,1] .
$$

Some important properties of $\theta\left(\cdot ; t_{0}, \theta_{0}, a, b\right), r\left(\cdot ; t_{0}, \theta_{0}, a, b\right), \Theta\left(\theta_{0}, a, b\right)$ and $X\left(\cdot ; \theta_{0}, a, b\right)$ are collected in the following theorem. 
Theorem 2.1 ([8]) Let $t_{0} \in[0,1]$ and $\theta_{0} \in \mathbb{R}$ be fixed. We have the following results.

(i) As mappings from $\left(L^{1}[0,1], w_{1}\right)^{2}$ to $\left(C[0,1],\|\cdot\|_{\infty}\right), \theta\left(\cdot ; t_{0}, \theta_{0}, a, b\right)$ and $r\left(\cdot ; t_{0}, \theta_{0}, a, b\right)$ are continuous. More precisely, if $a_{n} \stackrel{w_{1}}{\rightarrow} a_{0}$ and $b_{n} \stackrel{w_{1}}{\rightarrow} b_{0}$, then

$$
\begin{gathered}
\left\|\theta\left(\cdot ; t_{0}, \theta_{0}, a_{n}, b_{n}\right)-\theta\left(\cdot ; t_{0}, \theta_{0}, a_{0}, b_{0}\right)\right\|_{\infty} \rightarrow 0, \\
\left\|r\left(\cdot ; t_{0}, \theta_{0}, a_{n}, b_{n}\right)-r\left(\cdot ; t_{0}, \theta_{0}, a_{0}, b_{0}\right)\right\|_{\infty} \rightarrow 0,
\end{gathered}
$$

as $n \rightarrow \infty$.

(ii) The functional $\left(L^{1}[0,1], w_{1}\right)^{2} \rightarrow \mathbb{R},(a, b) \mapsto \Theta\left(\theta_{0}, a, b\right)$ is continuous. More precisely, if $a_{n} \stackrel{w_{1}}{\rightarrow} a_{0}$ and $b_{n} \stackrel{w_{1}}{\rightarrow} b_{0}$, then $\Theta\left(\theta_{0}, a_{n}, b_{n}\right) \rightarrow \Theta\left(\theta_{0}, a_{0}, b_{0}\right)$ as $n \rightarrow \infty$.

(iii) The functional $\left(L^{1}[0,1],\|\cdot\|_{1}\right)^{2} \rightarrow \mathbb{R},(a, b) \mapsto \Theta\left(\theta_{0}, a, b\right)$ is continuously differentiable in the sense of Fréchet. The differentials of $\Theta\left(\theta_{0}, a, b\right)$ at $a$ and $b$, denoted, respectively, by $\partial_{a} \Theta\left(\theta_{0}, a, b\right)$ and $\partial_{b} \Theta\left(\theta_{0}, a, b\right)$, are the following mappings:

$$
\begin{aligned}
& \partial_{a} \Theta\left(\theta_{0}, a, b\right)=X_{+}^{p}\left(\cdot ; \theta_{0}, a, b\right) \in\left(C[0,1],\|\cdot\|_{\infty}\right) \subset\left(L^{1},\|\cdot\|_{1}\right)^{*}, \\
& \partial_{b} \Theta\left(\theta_{0}, a, b\right)=X_{-}^{p}\left(\cdot ; \theta_{0}, a, b\right) \in\left(C[0,1],\|\cdot\|_{\infty}\right) \subset\left(L^{1},\|\cdot\|_{1}\right)^{*},
\end{aligned}
$$

where $\left(L^{1},\|\cdot\|_{1}\right)^{*}$ is the dual space of $\left(L^{1},\|\cdot\|_{1}\right)$. Moreover, as mappings from $\left(L^{1}[0,1], w_{1}\right)^{2}$ to $\left(C[0,1],\|\cdot\|_{\infty}\right)$, both $\partial_{a} \Theta\left(\theta_{0}, a, b\right)$ and $\partial_{b} \Theta\left(\theta_{0}, a, b\right)$ are continuous.

Remark 2.2 Let $\theta_{0} \in \mathbb{R}$ and $a_{i}, b_{i} \in L^{1}[0,1], i=1$, 2. If $a_{1} \leq a_{2}$ and $b_{1} \leq b_{2}$, then it follows from formulations (2.6) and (2.7) that

$$
\theta\left(1 ; 0, \theta_{0}, a_{1}, b_{1}\right) \leq \theta\left(1 ; 0, \theta_{0}, a_{2}, b_{2}\right) .
$$

\section{Fučík spectrum for Dirichlet problems: $\Pi_{p}^{D}(a, b)$}

\subsection{Decomposition of $\Pi_{p}^{D}(a, b)$}

Given a pair of weights $a, b \in L^{1}[0,1]$, the (Dirichlet type) Fučík spectrum $\Pi_{p}^{D}(a, b)$ is defined as the set of those $(\lambda, \mu) \in \mathbb{R}^{2}$ such that system (1.2)-(1.3) has non-trivial solutions. Let

$$
\phi_{p}\left(x^{\prime}\right)=-y \text {. }
$$

In the $p$-polar coordinates (2.2), equation (1.2) is equivalent to the following two equations:

$$
\begin{aligned}
& \theta^{\prime}=\lambda a(t)\left(\cos _{p} \theta\right)_{+}^{p}+\mu b(t)\left(\cos _{p} \theta\right)_{-}^{p}+(p-1)\left|\sin _{p} \theta\right|^{p^{*}}, \\
& (\log r)^{\prime}=\frac{p}{2}\left((\lambda a(t)-1)\left(\cos _{p} \theta\right)_{+}^{p-1}+(\mu b(t)-1)\left(\cos _{p} \theta\right)_{-}^{p-1}\right) \phi_{p^{*}}\left(\sin _{p} \theta\right) .
\end{aligned}
$$

Compared with equations (2.3) and (2.4), the pair of weights $a$ and $b$ are now replaced by $\lambda a$ and $\mu b$, respectively. Since the right-hand side of equation (3.2) is $2 \pi_{p}$-periodic in $\theta$, one has

$$
\theta\left(t ; t_{0}, \theta_{0}+2 l \pi_{p}, \lambda a, \mu b\right)=\theta\left(t ; t_{0}, \theta_{0}, \lambda a, \mu b\right)+2 l \pi_{p}, \quad \forall t \in[0,1]
$$

for any $t_{0} \in[0,1], \theta_{0} \in \mathbb{R}$ and $l \in \mathbb{Z}$. One can also check that

$$
\theta\left(t ; 0, \frac{\pi_{p}}{2}, \lambda a, \mu b\right)=\theta\left(t ; 0,-\frac{\pi_{p}}{2}, \mu b, \lambda a\right)+\pi_{p} .
$$


Suppose $x(t)$ is an eigenfunction of system (1.2)-(1.3) associated with $(\lambda, \mu) \in \Pi_{p}^{D}(a, b)$. By equation (2.2), the corresponding solution of equation (3.2), $\theta(t):=\theta\left(t ; 0,-\frac{\pi_{p}}{2}, \lambda a, \mu b\right)$, satisfies

$$
\theta(0)=-\frac{\pi_{p}}{2}+l \pi_{p} \quad \text { and } \quad \theta(1)=-\frac{\pi_{p}}{2}+(l+k) \pi_{p}
$$

for some $l, k \in \mathbb{Z}$. Due to equation (3.4), we may restrict $\theta(0) \in\left[-\frac{\pi_{p}}{2}, \frac{3 \pi_{p}}{2}\right)$. In other words, we may assume that $l \in\{0,1\}$ and hence $\theta(0)=-\frac{\pi_{p}}{2}$ or $\theta(0)=\frac{\pi_{p}}{2}$. Moreover, it follows from the quasi-monotonicity result in Lemma 2.2 that $k>0$. We distinguish two cases: $x^{\prime}(0)>0$ or $x^{\prime}(0)<0$. If $x^{\prime}(0)>0$, then it follows from equation (3.1) that $y(0)=-\phi_{p}\left(x^{\prime}(0)\right)<0$. By equation (2.2), we have $\sin _{p} \theta(0)<0$, and hence $l=0$ and $\theta(0)=-\frac{\pi_{p}}{2}$. Let

$$
W_{k}^{D}(a, b):=\left\{(\lambda, \mu) \in \mathbb{R}^{2}: \theta\left(1 ; 0,-\frac{\pi_{p}}{2}, \lambda a, \mu b\right)=k \pi_{p}-\frac{\pi_{p}}{2}\right\}, \quad \forall k \geq 1 .
$$

Now equation (3.6) tells us that $(\lambda, \mu) \in W_{k}^{D}(a, b)$. In fact, the subscript $k$ is related to the number of zeroes of $x(t)$ on $[0,1]$. By Lemma 2.2, the equation

$$
\theta(t)=-\frac{\pi_{p}}{2}+m \pi_{p}, \quad m \in \mathbb{Z}
$$

has a solution $t_{m} \in[0,1]$ if and only if $0 \leq m \leq k$, and

$$
0=t_{0}<t_{1}<\cdots<t_{k-1}<t_{k}=1 .
$$

By equation (2.2), we see that $x(t)$ has exactly $k+1$ zeroes in $[0,1]$. Similarly, if $x^{\prime}(0)<0$ and $x(t)$ has exactly $k+1$ zeroes in $[0,1]$, then $l=1, \theta(0)=\frac{\pi_{p}}{2}$ and $(\lambda, \mu) \in \widetilde{W}_{k}^{D}(a, b)$, where

$$
\widetilde{W}_{k}^{D}(a, b):=\left\{(\lambda, \mu) \in \mathbb{R}^{2}: \theta\left(1 ; 0, \frac{\pi_{p}}{2}, \lambda a, \mu b\right)=k \pi_{p}+\frac{\pi_{p}}{2}\right\}, \quad \forall k \geq 1
$$

Till now, we have proved that

$$
\Pi_{p}^{D}(a, b) \subset \bigcup_{k \in \mathbb{Z}, k \geq 1}\left(W_{k}^{D}(a, b) \cup \widetilde{W}_{k}^{D}(a, b)\right) .
$$

Conversely, let us show that

$$
\Pi_{p}^{D}(a, b) \supset \bigcup_{k \in \mathbb{Z}, k \geq 1}\left(W_{k}^{D}(a, b) \cup \widetilde{W}_{k}^{D}(a, b)\right)
$$

Suppose $(\lambda, \mu) \in W_{k}^{D}(a, b)$ for some $k \geq 1$. Then $\theta(t)=\theta\left(t ; 0,-\frac{\pi_{p}}{2}, \lambda a, \mu b\right)$ satisfies

$$
\theta(0)=-\frac{\pi_{p}}{2} \quad \text { and } \quad \theta(1)=-\frac{\pi_{p}}{2}+k \pi_{p}
$$

For this specific $\theta(t)$, take a non-trivial solution $r(t)$ of equation (3.3). Then we can construct a function $x(t)=r(t)^{2 / p} \cos _{p} \theta(t)$, which is a solution of equation (1.2) with exactly $k+1$ zeroes on $[0,1]$. Particularly, $x(0)=x(1)=0$. Thus $(\lambda, \mu) \in \Pi_{p}^{D}(a, b)$, and hence 
$W_{k}^{D}(a, b) \subset \Pi_{p}^{D}(a, b)$. Furthermore, we have

$$
y(0)=r^{2 / p^{*}}(0) \sin _{p} \theta(0)=r^{2 / p^{*}}(0) \sin _{p}\left(-\frac{\pi_{p}}{2}\right)<0,
$$

and hence

$$
x^{\prime}(0)=-\phi_{p^{*}}(y(0))>0 .
$$

Similarly, if $(\lambda, \mu) \in \widetilde{W}_{k}^{D}(a, b)$ for some $k \geq 1$, then $(\lambda, \mu) \in \Pi_{p}^{D}(a, b)$ and any associating eigenfunction $x(t)$ satisfies $x^{\prime}(0)<0$ and has exactly $k+1$ zeroes in $[0,1]$.

Combining the previous arguments, we can conclude that.

Theorem 3.1 Let $a, b \in L^{1}[0,1]$. The Fuč́l spectrum $\Pi_{p}^{D}(a, b)$ can be decomposed as

$$
\Pi_{p}^{D}(a, b)=\bigcup_{k \in \mathbb{Z}, k \geq 1}\left(W_{k}^{D}(a, b) \cup \widetilde{W}_{k}^{D}(a, b)\right) .
$$

Moreover, the following characterization on $W_{k}^{D}(a, b)$ and $\widetilde{W}_{k}^{D}(a, b)$ holds.

(i) $(\lambda, \mu) \in W_{k}^{D}(a, b), k \geq 1 \Longleftrightarrow$ any eigenfunction $x(t)$ associated with $(\lambda, \mu)$ satisfies $x^{\prime}(0)>0, x(0)=x(1)=1$ and $x(t)$ has precisely $k-1$ zeroes in $(0,1)$.

(ii) $(\lambda, \mu) \in \widetilde{W}_{k}^{D}(a, b), k \geq 1 \Longleftrightarrow$ any eigenfunction $x(t)$ associated with $(\lambda, \mu)$ satisfies $x^{\prime}(0)<0, x(0)=x(1)=1$ and $x(t)$ and has precisely $k-1$ zeroes in $(0,1)$.

By equation (3.5), the set $\widetilde{W}_{k}^{D}(a, b)$ defined as in equation (3.8) can be rewritten as

$$
\widetilde{W}_{k}^{D}(a, b)=\left\{(\lambda, \mu) \in \mathbb{R}^{2}: \theta\left(1 ; 0,-\frac{\pi_{p}}{2}, \mu b, \lambda a\right)=-\frac{\pi_{p}}{2}+k \pi_{p}\right\}, \quad \forall k \geq 1 .
$$

Thus

$$
(\lambda, \mu) \in \widetilde{W}_{k}^{D}(a, b) \Longleftrightarrow(\mu, \lambda) \in W_{k}^{D}(b, a) .
$$

In other words, $\widetilde{W}_{k}^{D}(a, b)$ is symmetric to $W_{k}^{D}(b, a)$ about the line $\lambda=\mu$. For this reason, essentially we need only to characterize those sets $W_{k}^{D}(a, b)$.

In Section 3.4, we will see that $W_{1}^{D}(a, b)$ is made up of straight lines which are in connection with $\lambda_{1}^{D}(a)$ and $\lambda_{-1}^{D}(a)$, the Dirichlet eigenvalues of $p$-Laplacian with the weight $a$. See Theorem 3.2.

For those sets $W_{k}^{D}(a, b), k \geq 2$, it is easy to check that

$$
\begin{aligned}
(\lambda, \mu) \in W_{k}^{D}(a, b) & \Longleftrightarrow(-\lambda, \mu) \in W_{k}^{D}(-a, b), \\
(\lambda, \mu) \in W_{k}^{D}(a, b) & \Longleftrightarrow(-\lambda,-\mu) \in W_{k}^{D}(-a,-b), \\
(\lambda, \mu) \in W_{k}^{D}(a, b) & \Longleftrightarrow(\lambda,-\mu) \in W_{k}^{D}(a,-b) .
\end{aligned}
$$

Therefore we need only to focus our study on the subset

$$
\Gamma_{k}^{D}(a, b):=W_{k}^{D}(a, b) \cap\left(\mathbb{R}_{+} \times \mathbb{R}_{+}\right), \quad k \geq 2,
$$


where $\mathbb{R}_{+}=[0,+\infty)$. In Section 3.4, for each $k \geq 2$ we will show that $\Gamma_{k}^{D}(a, b)$ is either an empty set or a continuous, strictly decreasing, hyperbolic like curve with a horizontal asymptotic line and a vertical asymptotic line.

With the help of half-eigenvalues of the $p$-Laplacian with a pair of weights, we can determined whether $\Gamma_{k}^{D}(a, b)$ is an empty set or not. Using eigenvalues of the $p$-Laplacian with a weight, we can roughly locate the hyperbolic like curve $\Gamma_{k}^{D}(a, b)$. For these reasons, we will give in the successive two subsections some useful characterization on eigenvalues and half-eigenvalues of the $p$-Laplacian with weights.

\subsection{Eigenvalues of $p$-Laplacian with an indefinite weight}

Given $a \in L^{1}[0,1]$, denote by $\vartheta(t)=\vartheta\left(t ; t_{0}, \vartheta_{0}, a\right)$ the solution of

$$
\theta^{\prime}=a(t)\left|\cos _{p} \theta\right|^{p}+(p-1)\left|\sin _{p} \theta\right|^{p^{*}}, \quad \text { a.e. } t \in[0,1],
$$

satisfying the initial value condition $\vartheta\left(t_{0}\right)=\vartheta_{0}$. Particularly, if $a(t) \equiv 1$, it follows from Lemma 2.1(vi) that equation (3.14) turns to be $\theta^{\prime} \equiv 1$, and hence

$$
\vartheta\left(t_{2} ; t_{1}, \vartheta_{0}, 1\right)=\vartheta_{0}+t_{2}-t_{1}, \quad \forall t_{1}, t_{2} \in[0,1], \vartheta_{0} \in \mathbb{R}
$$

Because the right-hand side of equation (3.14) is $\pi_{p}$-periodic in $\theta$, we have

$$
\vartheta\left(t ; t_{0}, \vartheta_{0}+k \pi_{p}, a\right)=\vartheta\left(t ; t_{0}, \vartheta_{0}, a\right)+k \pi_{p}
$$

for any $t_{0} \in[0,1], \vartheta_{0} \in \mathbb{R}$ and $k \in \mathbb{Z}$. Since equation (3.14) can also be rewritten as

$$
\theta^{\prime}=a(t)\left(\cos _{p} \theta\right)_{+}^{p}+a(t)\left(\cos _{p} \theta\right)_{-}^{p}+(p-1)\left|\sin _{p} \theta\right|^{p^{*}}, \quad \text { a.e. } t \in[0,1]
$$

using the notations in Section 2, we have

$$
\vartheta\left(t ; t_{0}, \vartheta_{0}, a\right)=\theta\left(t ; t_{0}, \vartheta_{0}, a, a\right)
$$

By Lemma 2.2, we see that $\vartheta\left(t ; t_{0}, \vartheta_{0}, a\right)$ is also quasi-monotonic in $t$.

Given $a \in L^{1}[0,1]$, denote by $\Sigma_{p}^{D}(a), \Sigma_{p}^{N}(a), \Sigma_{p}^{D N}(a)$ and $\Sigma_{p}^{N D}(a)$ the sets of $\lambda \in \mathbb{R}$ such that

$$
\left(\phi_{p}\left(x^{\prime}\right)\right)^{\prime}+\lambda a(t) \phi_{p}(x)=0, \quad \text { a.e. } t \in[0,1]
$$

has a non-trivial solution satisfying the Dirichlet boundary condition $x(0)=x(1)=0$, the Neumann boundary condition $x^{\prime}(0)=x^{\prime}(1)=0$, the Dirichlet-Neumann boundary condition $x(0)=x^{\prime}(1)=0$ and the Neumann-Dirichlet boundary condition $x^{\prime}(0)=x(1)=0$, respectively. Similar arguments as in Section 3.1 show that

$$
\begin{aligned}
& \Sigma_{p}^{D}=\bigcup_{k \in \mathbb{Z}, k \geq 1}\left\{\lambda \in \mathbb{R}: \vartheta\left(1 ; 0,-\frac{\pi_{p}}{2}, \lambda a\right)=-\frac{\pi_{p}}{2}+k \pi_{p}\right\}, \\
& \Sigma_{p}^{N}=\bigcup_{k \in \mathbb{Z}, k \geq 0}\left\{\lambda \in \mathbb{R}: \vartheta(1 ; 0,0, \lambda a)=k \pi_{p}\right\},
\end{aligned}
$$




$$
\begin{aligned}
& \Sigma_{p}^{D N}=\bigcup_{k \in \mathbb{Z}, k \geq 0}\left\{\lambda \in \mathbb{R}: \vartheta\left(1 ; 0,-\frac{\pi_{p}}{2}, \lambda a\right)=k \pi_{p}\right\}, \\
& \Sigma_{p}^{N D}=\bigcup_{k \in \mathbb{Z}, k \geq 1}\left\{\lambda \in \mathbb{R}: \vartheta(1 ; 0,0, \lambda a)=-\frac{\pi_{p}}{2}+k \pi_{p}\right\} .
\end{aligned}
$$

These spectra have been studied in [9]. Consider the function of $\lambda \in \mathbb{R}$ :

$$
\vartheta\left(1 ; 0,-\frac{\pi_{p}}{2}, \lambda a\right)=\theta\left(1 ; 0,-\frac{\pi_{p}}{2}, \lambda a, \lambda a\right)=\Theta\left(-\frac{\pi_{p}}{2}, \lambda a, \lambda a\right) .
$$

It follows from formulations (2.6) and (2.7) in Theorem 2.1 that

$$
\frac{d}{d \lambda} \vartheta\left(1 ; 0,-\frac{\pi_{p}}{2}, \lambda a\right)=\int_{0}^{1} X_{+}^{p}(t) a(t) d t+\int_{0}^{1} X_{-}^{p}(t) a(t) d t
$$

where $X(t)=X\left(t ;-\frac{\pi_{p}}{2}, \lambda a, \lambda a\right) \not \equiv 0$ satisfies

$$
\left(\phi_{p}\left(X^{\prime}\right)\right)^{\prime}+\lambda a(t) \phi_{p}\left(X_{+}\right)-\lambda a(t) \phi_{p}\left(X_{-}\right)=0, \quad \text { a.e. } t \in[0,1] .
$$

See equation (2.5) for the definition of $X(t)$. Then $X(t)$ is also a non-trivial solution of equation (3.17). Multiplying equation (3.20) by $X(t)$ and integrating over $[0,1]$, we have

$$
\begin{aligned}
& \lambda \int_{0}^{1} a(t) X_{+}^{p}(t) d t+\lambda \int_{0}^{1} a(t) X_{-}^{p}(t) d t \\
& \quad=-\int_{0}^{1}\left(\phi_{p}\left(X^{\prime}(t)\right)\right)^{\prime} X(t) d t \\
& \quad=-\left.X(t) \phi_{p}\left(X^{\prime}(t)\right)\right|_{t=0} ^{1}+\int_{0}^{1}\left|X^{\prime}(t)\right|^{p} d t .
\end{aligned}
$$

Substituting this into equation (3.19), for any $\lambda \neq 0$ we have

$$
\frac{d}{d \lambda} \vartheta\left(1 ; 0,-\frac{\pi_{p}}{2}, \lambda a\right)=-\left.\frac{1}{\lambda} \phi_{p}\left(X^{\prime}(t)\right) X(t)\right|_{t=0} ^{1}+\frac{1}{\lambda} \int_{0}^{1}\left|X^{\prime}(t)\right|^{p} d t
$$

If $\lambda \in \Sigma_{p}^{D}(a)$, then $X(t)$ becomes the associated eigenfunction of equation (3.17) satisfying $X(0)=X(1)=0$. In this case, the first item on the right-hand side of equation (3.21) equals 0 , and hence

$$
\pm\left.\frac{d}{d \lambda} \vartheta\left(1 ; 0,-\frac{\pi_{p}}{2}, \lambda a\right)\right|_{\lambda \in \Pi_{p}^{D}(a) \cap \mathbb{R}^{ \pm}}>0,
$$

where $\mathbb{R}^{+}=(0,+\infty)$ and $\mathbb{R}^{-}=(-\infty, 0)$. Similarly, we can obtain

$$
\begin{aligned}
& \pm\left.\frac{d}{d \lambda} \vartheta(1 ; 0,0, \lambda a)\right|_{\lambda \in \Pi_{p}^{N}(a) \cap \mathbb{R}^{ \pm}}>0, \\
& \pm\left.\frac{d}{d \lambda} \vartheta\left(1 ; 0,-\frac{\pi_{p}}{2}, \lambda a\right)\right|_{\lambda \in \Pi_{p}^{D N}(a) \cap \mathbb{R}^{ \pm}}>0, \\
& \pm\left.\frac{d}{d \lambda} \vartheta(1 ; 0,0, \lambda a)\right|_{\lambda \in \Pi_{p}^{N D}(a) \cap \mathbb{R}^{ \pm}}>0 .
\end{aligned}
$$


For any $k \geq 1$, it follows from equations (3.18) and (3.22) that

$$
\vartheta\left(1 ; 0,-\frac{\pi_{p}}{2}, \lambda a\right)=k \pi_{p}-\frac{\pi_{p}}{2}
$$

has at most one positive solution and one negative solution, denoted by $\lambda_{k}^{D}(a)$ and $\lambda_{-k}^{D}(a)$, respectively, if they exist. In other words, we have

$$
\begin{aligned}
& \lambda=\lambda_{k}^{D}(a), \quad k \geq 1 \quad \Longleftrightarrow \lambda>0 \quad \text { and } \vartheta\left(1 ; 0,-\frac{\pi_{p}}{2}, \lambda a\right)=k \pi_{p}-\frac{\pi_{p}}{2} ; \\
& \lambda=\lambda_{-k}^{D}(a), \quad k \geq 1 \Longleftrightarrow \lambda<0 \quad \text { and } \vartheta\left(1 ; 0,-\frac{\pi_{p}}{2}, \lambda a\right)=k \pi_{p}-\frac{\pi_{p}}{2} .
\end{aligned}
$$

It has been proved in [9] that $\vartheta(1 ; 0,0, \lambda a)=0$ has at most one nonzero solution, called the principal Neumann eigenvalue and denoted by $\lambda_{0}^{N}(a)$, if it exists. By equation (3.23) and the fact $\vartheta(1 ; 0,0 ; 0 \cdot a)=0$, we can deduce that $\vartheta(1 ; 0,0, \lambda a)=k \pi_{p}, k \geq 1$, has at most one positive solution and one negative solution, denoted by $\lambda_{k}^{N}(a)$ and $\lambda_{-k}^{N}(a)$ respectively, if they exist. In other words, we have

$$
\begin{aligned}
& \lambda=\lambda_{0}^{N}(a) \quad \Longleftrightarrow \quad \lambda \neq 0 \quad \text { and } \vartheta(1 ; 0,0, \lambda a)=0 ; \\
& \lambda=\lambda_{k}^{N}(a), \quad k \geq 1 \quad \Longleftrightarrow \quad \lambda>0 \quad \text { and } \vartheta(1 ; 0,0, \lambda a)=k \pi_{p} ; \\
& \lambda=\lambda_{-k}^{N}(a), \quad k \geq 1 \quad \Longleftrightarrow \quad \lambda<0 \quad \text { and } \vartheta(1 ; 0,0, \lambda a)=k \pi_{p} .
\end{aligned}
$$

For any $a \in L^{1}[0,1]$, use the notation $a \succ 0$ if $a(t) \geq 0$ for almost every $t \in[0,1]$ and $a(t)>0$ on a subset of $[0,1]$ of positive measure. Write $a \prec 0$ if $-a \succ 0$.

Lemma 3.1 ([9]) Let $a \in L^{1}[0,1]$. Then it is necessary that $0 \notin \Sigma_{p}^{D}(a)$.

(i) If $a \succ 0$, then $\Sigma_{p}^{D}(a)$ contains no negative eigenvalues, and it consists of a sequence of positive eigenvalues

$$
(0<) \lambda_{1}^{D}(a)<\lambda_{2}^{D}(a)<\cdots<\lambda_{k}^{D}(a)<\cdots(\rightarrow+\infty) .
$$

(ii) If $a \prec 0$, then $\Sigma_{p}^{D}(a)$ contains no positive eigenvalues, and it consists of a sequence of negative eigenvalues

$$
(0>) \lambda_{-1}^{D}(a)>\lambda_{-2}^{D}(a)>\cdots>\lambda_{-k}^{D}(a)>\cdots(\rightarrow-\infty) .
$$

(iii) If $a_{+} \succ 0$ and $a_{-} \succ 0$, then $\Sigma_{p}^{D}(a)$ contains both positive and negative eigenvalues, and it consists of a double sequence of eigenvalues

$$
(-\infty \leftarrow) \cdots<\lambda_{-k}^{D}(a)<\cdots<\lambda_{-1}^{D}(a)(<0<) \lambda_{1}^{D}(a)<\cdots<\lambda_{k}^{D}(a)<\cdots(\rightarrow+\infty) .
$$

Lemma $3.2([9])$ Let $a \in L^{1}[0,1]$. Then it is necessary that $0 \in \Sigma_{p}^{N}(a)$.

(i) If $a \succ 0$, then $\Sigma_{p}^{N}(a)$ contains no negative eigenvalues, and it consists of a sequence of non-negative eigenvalues

$$
0<\lambda_{1}^{N}(a)<\lambda_{2}^{N}(a)<\cdots<\lambda_{k}^{N}(a)<\cdots(\rightarrow+\infty) .
$$

The principal eigenvalue $\lambda_{0}^{N}(a)$ does not exist in this case. 
(ii) If $a \prec 0$, then $\Sigma_{p}^{N}(a)$ contains no positive eigenvalues, and it consists of a sequence of non-positive eigenvalues

$$
0>\lambda_{-1}^{N}(a)>\lambda_{-2}^{N}(a)>\cdots>\lambda_{-k}^{N}(a)>\cdots(\rightarrow-\infty) .
$$

The principal eigenvalue $\lambda_{0}^{N}(a)$ does not exist in this case.

(iii) If $a_{ \pm}>0$ and $\int_{0}^{1} a(t) d t<0$, then $\Sigma_{p}^{N}(a)$ contains both positive and negative eigenvalues, and it consists of a double sequence of eigenvalues

$$
\begin{aligned}
(-\infty \leftarrow) \cdots & <\lambda_{-k}^{N}(a)<\cdots<\lambda_{-1}^{N}(a)<0<\lambda_{0}^{N}(a) \\
& <\lambda_{1}^{N}(a)<\cdots<\lambda_{k}^{N}(a)<\cdots(\rightarrow+\infty) .
\end{aligned}
$$

The principal eigenvalue $\lambda_{0}^{N}(a)$ is positive in this case.

(iv) If $a_{ \pm}>0$ and $\int_{0}^{1} a(t) d t>0$, then $\Sigma_{p}^{N}(a)$ consists of $a$ double sequence of eigenvalues

$$
\begin{aligned}
(-\infty \leftarrow) \cdots & <\lambda_{-k}^{N}(a)<\cdots<\lambda_{-1}^{N}(a)<\lambda_{0}^{N}(a)<0 \\
& <\lambda_{1}^{N}(a)<\cdots<\lambda_{k}^{N}(a)<\cdots(\rightarrow+\infty) .
\end{aligned}
$$

The principal eigenvalue $\lambda_{0}^{N}(a)$ is negative in this case.

(v) If $a_{ \pm} \succ 0$ and $\int_{0}^{1} a(t) d t=0$, then $\Sigma_{p}^{N}(a)$ consists of a double sequence of eigenvalues

$$
\begin{aligned}
(-\infty \leftarrow) \cdots & <\lambda_{-k}^{N}(a)<\cdots<\lambda_{-1}^{N}(a)<0 \\
& <\lambda_{1}^{N}(a)<\cdots<\lambda_{k}^{N}(a)<\cdots(\rightarrow+\infty) .
\end{aligned}
$$

The principal eigenvalue $\lambda_{0}^{N}(a)$ does not exist in this case.

The following lemma reveals, to some extent, the essential reason of the existence of positive eigenvalues.

Lemma 3.3 Assume that $a \in L^{1}[0,1], 0 \leq t_{1}<t_{2} \leq 1, \vartheta_{0} \leq k \pi_{p}, \theta_{0} \leq k \pi_{p}-\frac{\pi_{p}}{2}$, and $k \in \mathbb{Z}$. Denote the indicator function of the subset $\left[t_{1}, t_{2}\right]$ of the set $[0,1]$ by $1_{\left[t_{1}, t_{2}\right]}$. Then

$$
\begin{aligned}
& a_{+} \cdot 1_{\left[t_{1}, t_{2}\right]}>0 \quad \Longleftrightarrow \quad \exists \lambda_{*}>0, \quad \text { s.t. } \vartheta\left(t_{2} ; t_{1}, \vartheta_{0}, \lambda_{*} a\right)=k \pi_{p}+\frac{\pi_{p}}{2} ; \\
& a_{+} \cdot 1_{\left[t_{1}, t_{2}\right]}>0 \quad \Longleftrightarrow \quad \exists \lambda^{*}>0, \quad \text { s.t. } \vartheta\left(t_{2} ; t_{1}, \theta_{0}, \lambda^{*} a\right)=k \pi_{p} .
\end{aligned}
$$

Proof We only prove equation (3.32), and equation (3.31) can be proved similarly.

Write $f(\lambda)=\vartheta\left(t_{2} ; t_{1}, \theta_{0}, \lambda a\right)$ for simplicity.

If $a_{+} \cdot 1_{\left[t_{1}, t_{2}\right]} \succ 0$, by similar arguments as in [9, Lemma 3.4] (see also Lemma 3.5) we have

$$
\lim _{\lambda \rightarrow+\infty} f(\lambda)=+\infty
$$

Let $\lambda=0$ in equation (3.2) and we get the equation

$$
\theta^{\prime}=(p-1)\left|\sin _{p} \theta\right|^{p^{*}}
$$


which has equilibria $\theta=k \pi_{p}, k \in \mathbb{Z}$. Because $\theta_{0} \leq k \pi_{p}-\frac{\pi_{p}}{2}<k \pi_{p}$, we get

$$
f(0)=\vartheta\left(t_{2} ; t_{1}, \theta_{0}, 0 \cdot a\right)<k \pi_{p} .
$$

Therefore there must exist $\lambda^{*}>0$ such that $f\left(\lambda^{*}\right)=k \pi_{p}$.

On the other hand, suppose that $a_{+} \cdot 1_{\left[t_{1}, t_{2}\right]}=0$, namely, $a(t) \leq 0$ for almost every $t \in\left[t_{1}, t_{2}\right]$. If $\lambda>0$, then $\lambda a(t)<1$ for almost every $t \in\left[t_{1}, t_{2}\right]$. Now it follows from the comparison theorem, equation (3.15), and Remark 2.1 that

$$
f(\lambda)<\vartheta\left(t_{2} ; t_{1}, \theta_{0}, 1\right)=\theta_{0}+t_{2}-t_{1}<k \pi_{p},
$$

completing the proof of equation (3.32).

In the rest of this subsection, we aim to reveal some quasi-monotonicity property of $\vartheta\left(t_{1} ; t_{0}, \vartheta_{0}, \lambda a\right)$ in $\lambda$, which will play an important role in analyzing the structure of the Fučík spectra $\Pi_{p}^{D / N}(a, b)$.

Using equation (3.18), the characterization on $\Sigma_{p}^{D}(a)$, we can rewritten equation (3.22) more precisely as

$$
\left.\begin{array}{l}
\vartheta\left(1 ; 0,-\frac{\pi_{p}}{2}, \lambda^{*} a\right)=-\frac{\pi_{p}}{2}+m \pi_{p}, \\
\pm \lambda^{*}>0, m \in \mathbb{Z}, m \geq 1
\end{array}\right\} \Rightarrow \pm\left.\frac{d}{d \lambda} \vartheta\left(1 ; 0,-\frac{\pi_{p}}{2}, \lambda a\right)\right|_{\lambda=\lambda^{*}}>0 .
$$

Furthermore, we have

$$
\begin{aligned}
& \left.\begin{array}{c}
\vartheta\left(1 ; 0,-\frac{\pi_{p}}{2}+k \pi_{p}, \lambda^{*} a\right)=-\frac{\pi_{p}}{2}+m \pi_{p} \\
\pm \lambda^{*}>0, k \in \mathbb{Z}, m \in \mathbb{Z}, m>k
\end{array}\right\} \\
& \Longrightarrow \pm\left.\frac{d}{d \lambda} \vartheta\left(1 ; 0,-\frac{\pi_{p}}{2}+k \pi_{p}, \lambda a\right)\right|_{\lambda=\lambda^{*}}>0,
\end{aligned}
$$

because it follows from equation (3.16) that

$$
\vartheta\left(1 ; 0,-\frac{\pi_{p}}{2}+k \pi_{p}, \lambda a\right)=\vartheta\left(1 ; 0,-\frac{\pi_{p}}{2}, \lambda a\right)+k \pi_{p}, \quad \forall k \in \mathbb{Z} .
$$

Though we have always been considering equations on the interval $t \in[0,1]$, similar results as in Theorem 2.1 still hold when the interval is replaced by any general interval. Thus equation (3.33) can also be generalized. In fact, for any $\alpha, \beta \in \mathbb{R}, \alpha<\beta$ and $a \in L^{1}[\alpha, \beta]$, we have

$$
\begin{aligned}
& \left.\begin{array}{c}
\vartheta\left(\beta ; \alpha,-\frac{\pi_{p}}{2}+k \pi_{p}, \lambda^{*} a\right)=-\frac{\pi_{p}}{2}+m \pi_{p}, \\
\pm \lambda^{*}>0, k \in \mathbb{Z}, m \in \mathbb{Z}, m>k
\end{array}\right\} \\
& \Longrightarrow \pm\left.\frac{d}{d \lambda} \vartheta\left(\beta ; \alpha,-\frac{\pi_{p}}{2}+k \pi_{p}, \lambda a\right)\right|_{\lambda=\lambda^{*}}>0 .
\end{aligned}
$$

Similar arguments can be applied to (3.23)-(3.25) to obtain results analogous to equation (3.34). We skip the proof and collect these results in the following lemma, which can be understood as the quasi-monotonicity of $\vartheta\left(t_{1} ; t_{0}, \vartheta_{0}, \lambda a\right)$ in $\lambda$. 
Lemma 3.4 Given $k \in \mathbb{Z}, \alpha, \beta \in \mathbb{R}, \alpha<\beta$, and $a \in L^{1}[\alpha, \beta]$, let

$$
\varphi(\lambda)=\vartheta\left(\beta ; \alpha, k \pi_{p}-\frac{\pi_{p}}{2}, \lambda a\right) \text { and } \psi(\lambda)=\vartheta\left(\beta ; \alpha, k \pi_{p}, \lambda a\right) .
$$

(i) If there exist $\lambda^{*}>0$ and an integer $m>k$ such that $\varphi\left(\lambda^{*}\right)=m \pi_{p}-\frac{\pi_{p}}{2}$, then

$\varphi^{\prime}\left(\lambda^{*}\right)>0$. Consequently, $\varphi(\lambda)>m \pi_{p}-\frac{\pi_{p}}{2}$ for any $\lambda \in\left(\lambda^{*},+\infty\right)$ and $\varphi(\lambda)<m \pi_{p}-\frac{\pi_{p}}{2}$ for any $\lambda \in\left(0, \lambda^{*}\right)$.

(ii) If there exist $\lambda^{*}>0$ and an integer $m \geq k$ such that $\varphi\left(\lambda^{*}\right)=m \pi_{p}$, then $\varphi^{\prime}\left(\lambda^{*}\right)>0$. Consequently, $\varphi(\lambda)>m \pi_{p}$ for any $\lambda \in\left(\lambda^{*},+\infty\right)$ and $\varphi(\lambda)<m \pi_{p}$ for any $\lambda \in\left(0, \lambda^{*}\right)$.

(iii) If there exist $\lambda^{*}>0$ and an integer $m \geq k$ such that $\psi\left(\lambda^{*}\right)=m \pi_{p}$, then $\psi^{\prime}\left(\lambda^{*}\right)>0$.

Consequently, $\psi(\lambda)>m \pi_{p}$ for any $\lambda \in\left(\lambda^{*},+\infty\right)$ and $\psi(\lambda)<m \pi_{p}$ for any $\lambda \in\left(0, \lambda^{*}\right)$.

(iv) If there exist $\lambda^{*}>0$ and an integer $m>k$ such that $\psi\left(\lambda^{*}\right)=m \pi_{p}-\frac{\pi_{p}}{2}$, then $\psi^{\prime}\left(\lambda^{*}\right)>0$. Consequently, $\psi(\lambda)>m \pi_{p}-\frac{\pi_{p}}{2}$ for any $\lambda \in\left(\lambda^{*},+\infty\right)$ and $\psi(\lambda)<m \pi_{p}-\frac{\pi_{p}}{2}$ for any $\lambda \in\left(0, \lambda^{*}\right)$.

\subsection{Half-eigenvalues of $p$-Laplacian with a pair of indefinite weights}

For any $a, b \in L^{1}[0,1]$, denote by $\mathcal{H}_{p}^{D}(a, b)$ and $\mathcal{H}_{p}^{N}(a, b)$ the sets of half-eigenvalues of the scalar $p$-Laplacian, namely, the sets of those $\lambda \in \mathbb{R}$ such that

$$
\left(\phi_{p}\left(x^{\prime}\right)\right)^{\prime}+\lambda a(t) \phi_{p}\left(x_{+}\right)-\lambda b(t) \phi_{p}\left(x_{-}\right)=0, \quad \text { a.e. } t \in[0,1]
$$

has a non-trivial solution satisfying the boundary conditions (1.3) and (1.4), respectively.

Based on the $p$-polar transformation (2.2) and the quasi-monotonicity results in Lemma 2.2, by similar arguments as in Section 3.1 we can show that

$$
\begin{aligned}
& \mathcal{H}_{p}^{D}(a, b)=\bigcup_{k \in \mathbb{Z}, k \geq 1}\left\{\lambda \in \mathbb{R}: \theta\left(1 ; 0,-\frac{\pi_{p}}{2}, \lambda a, \lambda b\right)=k \pi_{p}-\frac{\pi_{p}}{2}\right\}, \\
& \mathcal{H}_{p}^{N}(a, b)=\bigcup_{k \in \mathbb{Z}, k \geq 0}\left\{\lambda \in \mathbb{R}: \theta(1 ; 0,0, \lambda a, \lambda b)=k \pi_{p}\right\} .
\end{aligned}
$$

Applying the differentiability results (2.6) and (2.7) in Theorem 2.1, together with the Dirichlet boundary condition (1.3), by similar arguments as in Section 3.2 we can show that $\theta\left(1 ; 0,-\frac{\pi_{p}}{2}, \lambda a, \lambda b\right)$ is also quasi-monotonic in $\lambda$. More precisely, we have

$$
\begin{aligned}
& \left.\begin{array}{c}
\theta\left(1 ; 0,-\frac{\pi_{p}}{2}, \lambda^{*} a, \lambda^{*} b\right)=-\frac{\pi_{p}}{2}+k \pi_{p}, \\
\pm \lambda^{*}>0, k \in \mathbb{Z}, k \geq 1
\end{array}\right\} \\
& \Longrightarrow \pm\left.\frac{d}{d \lambda} \vartheta\left(1 ; 0,-\frac{\pi_{p}}{2}, \lambda a, \lambda b\right)\right|_{\lambda=\lambda^{*}}>0 .
\end{aligned}
$$

We also know that $\theta\left(1 ; 0,-\frac{\pi_{p}}{2}, 0 \cdot a, 0 \cdot b\right)<0$, because the equation

$$
\theta^{\prime}=(p-1)\left|\sin _{p}(\theta)\right|^{p^{*}}
$$

has equilibria $\theta=k \pi_{p}, k \in \mathbb{Z}$. Combining the quasi-monotonicity results in Lemma 2.2, we have

$$
-\frac{\pi_{p}}{2}<\theta\left(1 ; 0,-\frac{\pi_{p}}{2}, 0 \cdot a, 0 \cdot b\right)<0
$$


and hence $0 \notin \mathcal{H}_{p}^{D}(a, b)$. It follows from equations (3.35) and (3.36) that for any $k \geq 1$, the equation

$$
\theta\left(1 ; 0,-\frac{\pi_{p}}{2}, \lambda a, \lambda b\right)=k \pi_{p}-\frac{\pi_{p}}{2}
$$

has at most one positive solution and one negative solution, denoted, respectively, by $\Lambda_{k}^{D}(a, b)$ and $\Lambda_{-k}^{D}(a, b)$, if they exist. More precisely, we have

$$
\begin{aligned}
\lambda & =\Lambda_{k}^{D}(a, b), \quad k \geq 1 \\
& \Longleftrightarrow \lambda>0 \quad \text { and } \theta\left(1 ; 0,-\frac{\pi_{p}}{2}, \lambda a, \lambda b\right)=k \pi_{p}-\frac{\pi_{p}}{2} ; \\
\lambda & =\Lambda_{-k}^{D}(a, b), \quad k \geq 1 \\
& \Longleftrightarrow \quad \lambda<0 \quad \text { and } \theta\left(1 ; 0,-\frac{\pi_{p}}{2}, \lambda a, \lambda b\right)=k \pi_{p}-\frac{\pi_{p}}{2} .
\end{aligned}
$$

By equations (3.26), (3.27), and Lemma 2.2, we have $\Lambda_{ \pm 1}^{D}(a, b)=\lambda_{ \pm 1}^{D}(a)$. Some immediate results are

$$
\begin{aligned}
& \text { the existence of } \Lambda_{k+1}^{D}(a, b), \quad k \geq 1 \quad \Longrightarrow \quad \text { the existence of } \Lambda_{k}^{D}(a, b) \text {, } \\
& \text { the existence of } \Lambda_{-(k+1)}^{D}(a, b), \quad k \geq 1 \quad \Longrightarrow \quad \text { the existence of } \Lambda_{-k}^{D}(a, b) .
\end{aligned}
$$

Similarly, we have $\theta(1 ; 0,0,0 \cdot a, 0 \cdot b)=0$ and

$$
\left.\begin{array}{c}
\theta\left(1 ; 0,0, \lambda^{*} a, \lambda^{*} b\right)=k \pi_{p}, \\
\pm \lambda^{*}>0, k \in \mathbb{Z}, k \geq 0
\end{array}\right\} \Rightarrow \pm\left.\frac{d}{d \lambda} \vartheta(1 ; 0,0, \lambda a, \lambda b)\right|_{\lambda=\lambda^{*}}>0 .
$$

Thus the Neumann type half-eigenvalues $\Lambda_{ \pm k}^{N}(a, b), k \geq 1$, are defined as

$$
\begin{aligned}
& \lambda=\Lambda_{k}^{N}(a, b), \quad k \geq 1 \quad \Longleftrightarrow \quad \lambda>0 \quad \text { and } \quad \theta(1 ; 0,0, \lambda a, \lambda b)=k \pi_{p} ; \\
& \lambda=\Lambda_{-k}^{N}(a, b), \quad k \geq 1 \quad \Longleftrightarrow \quad \lambda<0 \quad \text { and } \quad \theta(1 ; 0,0, \lambda a, \lambda b)=k \pi_{p} .
\end{aligned}
$$

And the existence of $\Lambda_{ \pm(k+1)}^{N}(a, b), k \geq 1$, implies the existence of $\Lambda_{ \pm k}^{N}(a, b)$. By Lemma 2.2, the solution of $\theta(1 ; 0,0, \lambda a, \lambda b)=0$ is also that of $\theta(1 ; 0,0, \lambda a)=0$. Thus there may exist at most one principal Neumann half-eigenvalue $\Lambda_{0}^{N}(a, b)$, which is defined as

$$
\lambda=\Lambda_{0}^{N}(a, b) \Longleftrightarrow \lambda \neq 0 \quad \text { and } \quad \theta(1 ; 0,0, \lambda a, \lambda b)=0
$$

and $\Lambda_{0}^{N}(a, b)=\Lambda_{0}^{N}(a)$ by equation (3.28). Note that $\Lambda_{0}^{N}(a, b)$ may not exist even if $\Lambda_{ \pm 1}^{N}(a, b)$ exist.

It is easy to check that

$$
\Lambda_{-k}^{D}(a, b)=-\Lambda_{k}^{D}(-a,-b) \quad \text { and } \quad \Lambda_{-k}^{N}(a, b)=-\Lambda_{k}^{N}(-a,-b) .
$$

Essentially we need only to concern $\Lambda_{0}^{N}(a, b)$ and those positive half-eigenvalues $\Lambda_{k}^{D / N}(a$, $b), k \geq 1$. Now a natural question arises: for what kind of weights $a$ and $b$ do there exist no, finitely many, or infinitely many positive Dirichlet or Neumann type half-eigenvalues? 
Lemma 3.5 Assume that $a, b \in L^{1}[0,1]$ and $0 \leq t_{1}<t_{2} \leq 1$. If $a_{+} b_{+} \cdot 1_{\left[t_{1}, t_{2}\right]} \succ 0$, then

$$
\lim _{\lambda \rightarrow+\infty}\left(\theta\left(t_{2} ; t_{1}, \vartheta_{0}, \lambda a, \lambda b\right)-\vartheta_{0}\right)=+\infty
$$

uniformly in $\vartheta_{0} \in \mathbb{R}$.

Proof This lemma can be proved by similar argument as in the proof of Lemma 2.3 in [10], thus we skip the details.

Lemma 3.6 Suppose that $a, b \in L^{1}[0,1], 0 \leq t_{0}<t_{1}<\cdots<t_{k} \leq 1$, and there exist $\lambda_{i}>0$, $i=0,1, \ldots,\left\lfloor\frac{k-1}{2}\right\rfloor$, and $\mu_{i}>0, i=0,1, \ldots,\left\lfloor\frac{k}{2}\right\rfloor-1$, such that

$$
\begin{aligned}
& \vartheta\left(t_{2 i+1} ; t_{2 i}, 2 i \pi_{p}-\frac{\pi_{p}}{2}, \lambda_{i} a\right)=(2 i+1) \pi_{p}-\frac{\pi_{p}}{2}, \quad i=0,1, \ldots,\left\lfloor\frac{k-1}{2}\right\rfloor, \\
& \vartheta\left(t_{2 i+2} ; t_{2 i+1}, 2 i \pi_{p}+\frac{\pi_{p}}{2}, \mu_{i} b\right)=(2 i+1) \pi_{p}+\frac{\pi_{p}}{2}, \quad i=0,1, \ldots\left\lfloor\frac{k}{2}\right\rfloor-1 .
\end{aligned}
$$

Let $\lambda^{*} \geq \max \left\{\lambda_{i}: i=0,1, \ldots,\left\lfloor\frac{k-1}{2}\right\rfloor\right\}$ and $\mu^{*} \geq \max \left\{\mu_{i}: i=0,1, \ldots,\left\lfloor\frac{k}{2}\right\rfloor-1\right\}$. Then

$$
\theta\left(t_{k} ; t_{0},-\frac{\pi_{p}}{2}, \lambda^{*} a, \mu^{*} b\right) \geq k \pi_{p}-\frac{\pi_{p}}{2},
$$

and inequality (3.43) becomes an equality if and only if

$$
\lambda^{*}=\lambda_{i}, \quad \forall i=0,1, \ldots,\left\lfloor\frac{k-1}{2}\right\rfloor \quad \text { and } \quad \mu^{*}=\mu_{i}, \quad \forall i=0,1, \ldots,\left\lfloor\frac{k}{2}\right\rfloor-1 .
$$

Proof Let us write $\theta(t)=\theta\left(t ; t_{0},-\frac{\pi_{p}}{2}, \lambda^{*} a, \mu^{*} b\right)$ for simplicity.

Claim I: there exists $t_{1}^{*} \in\left(t_{0}, t_{1}\right]$ such that $\theta\left(t_{1}^{*}\right)=\frac{\pi_{p}}{2}$. If this is false, then it follows from Lemma 2.2 that

$$
\theta(t) \in\left(-\frac{\pi_{p}}{2}, \frac{\pi_{p}}{2}\right), \quad \forall t \in\left(t_{0}, t_{1}\right]
$$

and hence $\cos _{p} \theta(t)>0$ for any $t \in\left(t_{0}, t_{1}\right)$. Recall that $\theta(t)$ satisfies the ODE

$$
\theta^{\prime}(t)=\lambda^{*} a(t)\left(\cos _{p} \theta\right)_{+}^{p}+\mu^{*} b(t)\left(\cos _{p} \theta\right)_{-}^{p}+(p-1)\left|\sin _{p} \theta\right|^{p^{*}}, \quad \text { a.e. } t \in[0,1] .
$$

Then we can conclude that $\theta(t)$ also satisfies

$$
\theta^{\prime}(t)=\lambda^{*} a(t)\left|\cos _{p} \theta\right|^{p}+(p-1)\left|\sin _{p} \theta\right|^{p^{*}}
$$

on the interval $t \in\left[t_{0}, t_{1}\right]$. Thus we have

$$
\theta(t)=\vartheta\left(t ; t_{0},-\frac{\pi_{p}}{2}, \lambda^{*} a\right), \quad \forall t \in\left[t_{0}, t_{1}\right]
$$

Particularly, we get from equations (3.44) and (3.46)

$$
\vartheta\left(t_{1} ; t_{0},-\frac{\pi_{p}}{2}, \lambda^{*} a\right)=\theta\left(t_{1}\right)<\frac{\pi_{p}}{2} .
$$


On the other hand, let $i=0$ in equation (3.41), we get

$$
\vartheta\left(t_{1} ; t_{0},-\frac{\pi_{p}}{2}, \lambda_{0} a\right)=\frac{\pi_{p}}{2} .
$$

Since $\lambda^{*} \geq \lambda_{0}>0$, it follows from Lemma 3.4(i) that

$$
\vartheta\left(t_{1} ; t_{0},-\frac{\pi_{p}}{2}, \lambda^{*} a\right) \geq \frac{\pi_{p}}{2}
$$

a contradiction to equation (3.47). Thus there exists $t_{1}^{*} \in\left(t_{0}, t_{1}\right]$ such that $\theta\left(t_{1}^{*}\right)=\frac{\pi_{p}}{2}$, proving Claim I.

If $\lambda^{*}>\lambda_{0}$, we aim to show that $t_{1}^{*}<t_{1}$. If this is not true, then $t_{1}^{*}=t_{1}$, and one can check that equation (3.46) is still true. It follows from equations (3.46), (3.48), Lemma 3.4(i), and the fact $\lambda^{*}>\lambda_{0}$ that

$$
\theta\left(t_{1}^{*}\right)=\theta\left(t_{1}\right)=\vartheta\left(t_{1} ; t_{0},-\frac{\pi_{p}}{2}, \lambda^{*} a\right)>\frac{\pi_{p}}{2},
$$

a contradiction to $\theta\left(t_{1}^{*}\right)=\frac{\pi_{p}}{2}$.

Claim II: there exists $t_{2}^{*} \in\left(t_{1}^{*}, t_{2}\right]$ such that $\theta\left(t_{2}^{*}\right)=\frac{3 \pi_{p}}{2}$. If this is not true, then the quasimonotonicity of $\theta(t)$ in $t$ shows that

$$
\theta(t) \in\left(\frac{\pi_{p}}{2}, \frac{3 \pi_{p}}{2}\right), \quad \forall t \in\left(t_{1}^{*}, t_{2}\right]
$$

Thus $\theta(t)$ satisfies

$$
\theta^{\prime}(t)=\mu^{*} b(t)\left|\cos _{p} \theta\right|^{p}+(p-1)\left|\sin _{p} \theta\right|^{p^{*}}
$$

on the interval $t \in\left[t_{1}^{*}, t_{2}\right]$, and the initial value condition $\theta\left(t_{1}^{*}\right)=\frac{\pi_{p}}{2}$. Therefore

$$
\theta(t)=\vartheta\left(t ; t_{1}^{*}, \frac{\pi_{p}}{2}, \mu^{*} b\right), \quad \forall t \in\left[t_{1}^{*}, t_{2}\right]
$$

If $t_{1}^{*}=t_{1}$, similar arguments as in the proof of Claim I show the existence of $t_{2}^{*} \in\left(t_{1}^{*}, t_{2}\right]$ such that $\theta\left(t_{2}^{*}\right)=\frac{3 \pi_{p}}{2}$, and Claim II is proved. Moreover, if $\mu^{*}>\mu_{0}$, then $t_{2}^{*}<t_{2}$.

If $t_{1}^{*}<t_{1}$, then $\theta\left(t_{1}\right)>\frac{\pi_{p}}{2}$ by Lemma 2.2. We can improve the result in Claim II as the existence of $t_{2}^{*} \in\left(t_{1}^{*}, t_{2}\right)$ such that $\theta\left(t_{2}^{*}\right)=\frac{3 \pi_{p}}{2}$. If this result is not true, then

$$
\theta(t) \in\left(\frac{\pi_{p}}{2}, \frac{3 \pi_{p}}{2}\right), \quad \forall t \in\left(t_{1}^{*}, t_{2}\right)
$$

and we still have equations (3.49)-(3.50). Now both $\theta(t)$ and $\vartheta(t):=\vartheta\left(t ; t_{1}, \frac{\pi_{p}}{2}, \mu^{*} b\right)$ satisfy the same ODE (3.49) on the interval $t \in\left[t_{1}, t_{2}\right]$, while the initial values satisfy the condition

$$
\theta\left(t_{1}\right)>\frac{\pi_{p}}{2}=\vartheta\left(t_{1}\right)
$$


By case $i=0$ in equation (3.42) we have $\vartheta\left(t_{2}\right)=\frac{3 \pi_{p}}{2}$. Now the existence and uniqueness theorem for the first-order ODEs shows that

$$
\theta\left(t_{2}\right)>\vartheta\left(t_{2}\right)=\frac{3 \pi_{p}}{2}
$$

Since $\theta\left(t_{1}^{*}\right)=\frac{\pi_{p}}{2}$, there exists $t_{2}^{*} \in\left(t_{1}^{*}, t_{2}\right)$ such that $\theta\left(t_{2}^{*}\right)=\frac{3 \pi_{p}}{2}$.

Now we can conclude that Claim II is true. Moreover, if $\lambda^{*}>\lambda_{0}$ or $\mu^{*}>\lambda_{0}$, then $t_{2}^{*}<t_{2}$. Inductively, we can show that there exists $t_{k}^{*} \in\left(t_{k-1}^{*}, t_{k}\right]$ such that $\theta\left(t_{k}^{*}\right)=k \pi_{p}-\frac{\pi_{p}}{2}$. Moreover, we have $t_{k}^{*}<t_{k}$, if $\lambda^{*}>\lambda_{i_{0}}$ for some $i_{0} \in\left\{0,1, \ldots,\left\lfloor\frac{k-1}{2}\right\rfloor\right\}$, or $\mu^{*}>\mu_{j_{0}}$ for some $j_{0} \in\left\{0,1, \ldots,\left\lfloor\frac{k}{2}\right\rfloor-1\right\}$. And if $t_{k}^{*}<t_{k}$, it follows from Lemma 2.2 that $\theta\left(t_{k}\right)>k \pi_{p}-\frac{\pi_{p}}{2}$.

Finally, if $\lambda^{*}=\lambda_{i}, i=0,1, \ldots,\left\lfloor\frac{k-1}{2}\right\rfloor$, and $\mu^{*}=\mu_{i}, i=0,1, \ldots,\left\lfloor\frac{k}{2}\right\rfloor-1$, then it follows from equations (3.41) and (3.42) that $\theta\left(t_{k} ; t_{0},-\frac{\pi_{p}}{2}, \lambda^{*} a, \mu^{*} b\right)=k \pi_{p}-\frac{\pi_{p}}{2}$, completing the proof of the lemma.

Property 3.1 Given $a, b \in L^{1}[0,1]$, we have the following results:

(i) if $a \prec 0$, then any positive half-eigenvalues $\Lambda_{k}^{D}(a, b), k \geq 1$, does not exist;

(ii) if $a_{+} b_{+} \succ 0$, then all positive half-eigenvalues $\Lambda_{k}^{D}(a, b), k \geq 1$, exist;

(iii) $\Lambda_{k+1}^{D}(a, b)$ exists, $k \geq 1 \Longrightarrow$ both $\Lambda_{k}^{D}(a, b)$ and $\Lambda_{k}^{D}(b, a)$ exist;

(iv) both $\Lambda_{k}^{D}(a, b)$ and $\Lambda_{k}^{D}(b, a)$ exist, $k \geq 1 \Longrightarrow \Lambda_{k+1}^{D}(a, b)$ exists or $\Lambda_{k+1}^{D}(b, a)$ exists.

Proof (i) Assume that $a \prec 0$. If there exists a positive half-eigenvalue $\lambda^{*}=\Lambda_{k_{0}}^{D}(a, b), k_{0} \geq 1$, then

$$
\theta\left(1 ; 0,-\frac{\pi_{p}}{2}, \lambda^{*} a, \lambda^{*} b\right)=k_{0} \pi_{p}-\frac{\pi_{p}}{2}
$$

and hence there must exist $t_{1} \in(0,1]$ such that

$$
\theta\left(t_{1} ; 0,-\frac{\pi_{p}}{2}, \lambda^{*} a, \lambda^{*} b\right)=\frac{\pi_{p}}{2}
$$

It follows from Lemma 2.2 that

$$
\theta(t)=\theta\left(t ; 0,-\frac{\pi_{p}}{2}, \lambda^{*} a, \lambda^{*} b\right) \in\left(-\frac{\pi_{p}}{2}, \frac{\pi_{p}}{2}\right), \quad \forall t \in\left(0, t_{1}\right)
$$

and hence

$$
\theta\left(t ; 0,-\frac{\pi_{p}}{2}, \lambda^{*} a, \lambda^{*} b\right)=\vartheta\left(t ; 0,-\frac{\pi_{p}}{2}, \lambda^{*} a\right), \quad \forall t \in\left[0, t_{1}\right]
$$

Particularly, we get

$$
\vartheta\left(t_{1} ; 0,-\frac{\pi_{p}}{2}, \lambda^{*} a\right)=\theta\left(t_{1} ; 0,-\frac{\pi_{p}}{2}, \lambda^{*} a, \lambda^{*} b\right)=\frac{\pi_{p}}{2} .
$$

By Lemma 3.3, we have $a_{+} \cdot 1_{\left[0, t_{1}\right]} \succ 0$, a contradiction to the assumption $a \prec 0$. Consequently, there is not any positive half-eigenvalue $\Lambda_{k}^{D}(a, b), k \geq 1$, if $a \prec 0$.

(ii) This result follows immediately from equations (3.35), (3.36), and Lemma 3.5. 
(iii) Assume that $\lambda^{*}=\Lambda_{k+1}^{D}(a, b)$ exists and $k \geq 1$. The existence of $\Lambda_{k}^{D}(a, b)$ has been given in equation (3.39). We need only to prove the existence of $\Lambda_{k}^{D}(b, a)$. Take the following notations for simplicity:

$$
\theta(t):=\theta\left(t ; 0,-\frac{\pi_{p}}{2}, \lambda^{*} a, \lambda^{*} b\right), \quad \tilde{\theta}(t):=\theta\left(t ; 0,-\frac{\pi_{p}}{2}, \lambda^{*} b, \lambda^{*} a\right)
$$

By the definition of $\Lambda_{k+1}^{D}(a, b)$, we have $\theta(1)=(k+1) \pi_{p}-\frac{\pi_{p}}{2}$. By Lemma 2.2, there exist $0=t_{0}<t_{1}<\cdots<t_{k}<t_{k+1}=1$, such that

$$
\theta\left(t_{i}\right)=i \pi_{p}-\frac{\pi_{p}}{2}, \quad 0 \leq i \leq k+1
$$

and

$$
i \pi_{p}-\frac{\pi_{p}}{2}<\theta(t)<i \pi_{p}+\frac{\pi_{p}}{2}, \quad \forall t \in\left(t_{i}, t_{i+1}\right), 0 \leq i \leq k
$$

Therefore $\cos _{p} \theta(t)>(<) 0$ if $t \in\left(t_{i}, t_{i+1}\right)$ and $i$ is even (odd). Thus

$$
\begin{aligned}
& \theta(t)=\vartheta\left(t ; t_{i}, i \pi_{p}-\frac{\pi_{p}}{2}, \lambda^{*} a\right), \quad \forall t \in\left(t_{i}, t_{i+1}\right), 0 \leq i \leq k, i \text { is even, } \\
& \theta(t)=\vartheta\left(t ; t_{i}, i \pi_{p}-\frac{\pi_{p}}{2}, \lambda^{*} b\right), \quad \forall t \in\left(t_{i}, t_{i+1}\right), 0 \leq i \leq k, i \text { is odd. }
\end{aligned}
$$

We claim that

$$
\tilde{\theta}\left(t_{i}\right)>i \pi_{p}-\frac{3 \pi_{p}}{2}, \quad i=1,2, \ldots, k+1 .
$$

In fact, we have $\widetilde{\theta}\left(t_{1}\right)>-\frac{\pi_{p}}{2}$ by Lemma 2.2, proving equation (3.52) for the case $i=1$. To prove the case $i=2$, we assume on the contrary that $\tilde{\theta}\left(t_{2}\right) \leq \frac{\pi_{p}}{2}$, then

$$
-\frac{\pi_{p}}{2}<\tilde{\theta}(t)<\frac{\pi_{p}}{2}, \quad \forall t \in\left(t_{1}, t_{2}\right)
$$

and hence

$$
\widetilde{\theta}(t)=\vartheta\left(t ; t_{1}, \widetilde{\theta}\left(t_{1}\right), \lambda^{*} b\right), \quad \forall t \in\left(t_{1}, t_{2}\right)
$$

Letting $i=1$ in equation (3.51), we see that $\theta(t)$ and $\tilde{\theta}(t)$ satisfy the same ODE,

$$
\theta^{\prime}(t)=\lambda^{*} b(t)\left|\cos _{p} \theta\right|^{p}+(p-1)\left|\sin _{p} \theta\right|^{p^{*}}, \quad t \in\left[t_{1}, t_{2}\right] .
$$

Moreover, $\varphi(t):=\theta(t)-\pi_{p}$ is also a solution of equation (3.53). Since

$$
\varphi\left(t_{1}\right)=\theta\left(t_{1}\right)-\pi_{p}=-\frac{\pi_{p}}{2}<\tilde{\theta}\left(t_{1}\right)
$$

we obtain

$$
\widetilde{\theta}\left(t_{2}\right)>\varphi\left(t_{2}\right)=\theta\left(t_{2}\right)-\pi_{p}=\frac{\pi_{p}}{2},
$$


thus the assumption $\tilde{\theta}\left(t_{2}\right) \leq \frac{\pi_{p}}{2}$ is false, proving equation (3.52) for the case $i=2$. Inductively, we can prove equation (3.52).

Let $i=k+1$ in equation (3.52); we get

$$
\theta\left(1 ; 0,-\frac{\pi_{p}}{2}, \lambda^{*} b, \lambda^{*} a\right)=\widetilde{\theta}(1)>k \pi_{p}-\frac{\pi_{p}}{2}
$$

Combining with equation (3.36), we conclude that there must exist $\tilde{\lambda}=\Lambda_{k}^{D}(b, a) \in\left(0, \lambda^{*}\right)$ such that

$$
\theta\left(1 ; 0,-\frac{\pi_{p}}{2}, \tilde{\lambda} b, \tilde{\lambda} a\right)=k \pi_{p}-\frac{\pi_{p}}{2} .
$$

(iv) Suppose that both $\lambda^{*}=\Lambda_{k}^{D}(a, b)$ and $\lambda_{*}=\Lambda_{k}^{D}(b, a)$ exist and $k \geq 1$. We assume that $k=2 m+1, m \geq 0$. The case $k=2 m$ can be proved similarly.

Let us take the notations

$$
\theta(t):=\theta\left(t ; 0,-\frac{\pi_{p}}{2}, \lambda^{*} a, \lambda^{*} b\right) \text { and } \tilde{\theta}(t):=\theta\left(t ; 0,-\frac{\pi_{p}}{2}, \lambda_{*} b, \lambda_{*} a\right) .
$$

By the definition of half-eigenvalues in equation (3.37) and Lemma 2.2, there exist $0=t_{0}<$ $t_{1}<\cdots<t_{2 m}<t_{2 m+1}=1$ and $0=s_{0}<s_{1}<\cdots<s_{2 m}<s_{2 m+1}=1$, such that

$$
\begin{aligned}
& \theta\left(t_{i}\right)=i \pi_{p}-\frac{\pi_{p}}{2}, \quad i=0,1, \ldots, 2 m+1 ; \\
& \widetilde{\theta}\left(s_{i}\right)=i \pi_{p}-\frac{\pi_{p}}{2}, \quad i=0,1, \ldots, 2 m+1
\end{aligned}
$$

and

$$
\begin{aligned}
& \theta(t)=\vartheta\left(t ; t_{2 i}, 2 i \pi_{p}-\frac{\pi_{p}}{2}, \lambda^{*} a\right), \quad \forall t \in\left[t_{2 i}, t_{2 i+1}\right], 0 \leq i \leq m ; \\
& \theta(t)=\vartheta\left(t ; t_{2 i+1}, 2 i \pi_{p}+\frac{\pi_{p}}{2}, \lambda^{*} b\right), \quad \forall t \in\left[t_{2 i+1}, t_{2 i+2}\right], 0 \leq i \leq m-1 ; \\
& \widetilde{\theta}(t)=\vartheta\left(t ; s_{2 i}, 2 i \pi_{p}-\frac{\pi_{p}}{2}, \lambda_{*} b\right), \quad \forall t \in\left[s_{2 i}, s_{2 i+1}\right], 0 \leq i \leq m ; \\
& \widetilde{\theta}(t)=\vartheta\left(t ; s_{2 i+1}, 2 i \pi_{p}+\frac{\pi_{p}}{2}, \lambda_{*} a\right), \quad \forall t \in\left[s_{2 i+1}, s_{2 i+2}\right], 0 \leq i \leq m-1 .
\end{aligned}
$$

Particularly, we get

$$
\begin{aligned}
& \vartheta\left(t_{2 i+1} ; t_{2 i}, 2 i \pi_{p}-\frac{\pi_{p}}{2}, \lambda^{*} a\right)=2 i \pi_{p}+\frac{\pi_{p}}{2}, \quad 0 \leq i \leq m ; \\
& \vartheta\left(t_{2 i+2} ; t_{2 i+1}, 2 i \pi_{p}+\frac{\pi_{p}}{2}, \lambda^{*} b\right)=2 i \pi_{p}+\frac{3 \pi_{p}}{2}, \quad 0 \leq i \leq m-1 ; \\
& \vartheta\left(s_{2 i+1} ; s_{2 i}, 2 i \pi_{p}-\frac{\pi_{p}}{2}, \lambda_{*} b\right)=2 i \pi_{p}+\frac{\pi_{p}}{2}, \quad 0 \leq i \leq m ; \\
& \vartheta\left(s_{2 i+2} ; s_{2 i+1}, 2 i \pi_{p}+\frac{\pi_{p}}{2}, \lambda_{*} a\right)=2 i \pi_{p}+\frac{\pi_{p}}{2}, \quad 0 \leq i \leq m-1 .
\end{aligned}
$$


Let $i=m$ in equations (3.54) and (3.56). We get from Lemma 3.3

$$
a_{+} \cdot 1_{\left[t_{2 m}, 1\right]} \succ 0 \text { and } \quad b_{+} \cdot 1_{\left[s_{2 m}, 1\right]} \succ 0 .
$$

Without loss of generality, we may assume that $t_{2 m} \leq s_{2 m}$. We need only to distinguish three cases in the following.

Case 1. $a_{+} \cdot 1_{\left[t_{2 m}, s_{2 m}\right]} \succ 0$ and $b_{+} \cdot 1_{\left[s_{2 m}, 1\right]} \succ 0$. In this case, it follows from Lemma 3.3 that there exist $\lambda_{1}>0$ and $\lambda_{2}>0$ such that

$$
\begin{aligned}
& \vartheta\left(s_{2 m} ; t_{2 m}, 2 m \pi_{p}-\frac{\pi_{p}}{2}, \lambda_{1} a\right)=(2 m+1) \pi_{p}-\frac{\pi_{p}}{2}, \\
& \vartheta\left(1 ; s_{2 m} ; 2 m \pi_{p}+\frac{\pi_{p}}{2}, \lambda_{2} b\right)=(2 m+2) \pi_{p}-\frac{\pi_{p}}{2} .
\end{aligned}
$$

Let $\bar{\lambda}=\max \left\{\lambda^{*}, \lambda_{1}, \lambda_{2}\right\}$. Combining equations (3.54), (3.55), and the above two conditions, we get from Lemma 3.6

$$
\theta\left(1 ; 0,-\frac{\pi_{p}}{2}, \bar{\lambda} a, \bar{\lambda} b\right) \geq(2 m+2) \pi_{p}-\frac{\pi_{p}}{2}=(k+1) \pi_{p}-\frac{\pi_{p}}{2},
$$

and hence $\Lambda_{k+1}^{D}(a, b)$ exists.

Case 2. $a_{+} \cdot 1_{\left[t_{2 m}, s_{2 m}\right]}=0$ and $a_{+} b_{+} \cdot 1_{\left[s_{2 m}, 1\right]} \succ 0$. In this case, we have $a_{+} b_{+} \succ 0$. And it follows from the result (ii) in this property that both $\Lambda_{k+1}^{D}(a, b)$ and $\Lambda_{k+1}^{D}(b, a)$ exist.

Case 3. $a_{+} \cdot 1_{\left[t_{2 m}, s_{2 m}\right]}=0$ and $a_{+} b_{+} \cdot 1_{\left[s_{2 m}, 1\right]}=0$. In this case, since $a_{+} \cdot 1_{\left[t_{2 m}, s_{2 m}\right]}=0$, the condition (3.58) can be written as

$$
a_{+} \cdot 1_{\left[s_{2 m}, 1\right]} \succ 0 \text { and } \quad b_{+} \cdot 1_{\left[s_{2 m}, 1\right]} \succ 0 \text {. }
$$

Because $a_{+} b_{+} \cdot 1_{\left[s_{2 m}, 1\right]}=0$, there must exist $\tau \in\left(s_{2 m}, 1\right)$, such that

$$
a_{+} \cdot 1_{\left[s_{2 m}, \tau\right]} \succ 0 \text { and } \quad b_{+} \cdot 1_{[\tau, 1]} \succ 0
$$

or

$$
b_{+} \cdot 1_{\left[s_{2 m}, \tau\right]} \succ 0 \text { and } a_{+} \cdot 1_{[\tau, 1]} \succ 0 .
$$

Then we can apply similar arguments as in Case 1 to get the existence of $\Lambda_{k+1}^{D}(a, b)$ if equation (3.59) holds, and the existence of $\Lambda_{k+1}^{D}(b, a)$ if equation (3.59) holds.

Corollary 3.1 Let $a, b \in L^{1}[0,1]$ and denote $\mathbb{R}^{+}=(0,+\infty)$. One of the following three cases must occur.

(i) $\mathcal{H}_{p}^{D}(a, b) \cap \mathbb{R}^{+}=\emptyset$ and $\mathcal{H}_{p}^{D}(b, a) \cap \mathbb{R}^{+}=\emptyset$.

(ii) $\mathcal{H}_{p}^{D}(a, b) \cap \mathbb{R}^{+}=\bigcup_{k \geq 1} \Lambda_{k}^{D}(a, b)$ and $\mathcal{H}_{p}^{D}(b, a) \cap \mathbb{R}^{+}=\bigcup_{k \geq 1} \Lambda_{k}^{D}(b, a)$.

(iii) There exists $k_{0} \geq 1$ such that either

$$
\mathcal{H}_{p}^{D}(a, b) \cap \mathbb{R}^{+}=\bigcup_{1 \leq k \leq k_{0}} \Lambda_{k}^{D}(a, b), \quad \mathcal{H}_{p}^{D}(b, a) \cap \mathbb{R}^{+}=\bigcup_{1 \leq k \leq k_{0}-1} \Lambda_{k}^{D}(b, a)
$$


or

$$
\mathcal{H}_{p}^{D}(a, b) \cap \mathbb{R}^{+}=\bigcup_{1 \leq k \leq k_{0}-1} \Lambda_{k}^{D}(a, b), \quad \mathcal{H}_{p}^{D}(b, a) \cap \mathbb{R}^{+}=\bigcup_{1 \leq k \leq k_{0}} \Lambda_{k}^{D}(b, a) .
$$

Applying Lemmas 3.3 and 3.6, one can verify the following three examples.

Example 3.1 Suppose $a, b \in L^{1}[0,1], 0=t_{0}<t_{1}<\cdots<t_{2 n}<t_{2 n+1}=1, n \geq 0$, and

$$
\begin{array}{lll}
a_{+} \cdot 1_{\left[t_{k}, t_{k+1}\right]} \succ 0, & b_{+} \cdot 1_{\left[t_{k}, t_{k+1}\right]}=0, & k=0,2,4, \ldots, 2 n, \\
a_{+} \cdot 1_{\left[t_{k}, t_{k+1}\right]}=0, & b_{+} \cdot 1_{\left[t_{k}, t_{k+1}\right]} \succ 0, & k=1,3,5, \ldots, 2 n-1 .
\end{array}
$$

Then $\mathcal{H}_{p}^{D}(a, b) \cap \mathbb{R}^{+}=\bigcup_{1 \leq k \leq 2 n+1} \Lambda_{k}^{D}(a, b)$ and $\mathcal{H}_{p}^{D}(b, a) \cap \mathbb{R}^{+}=\bigcup_{1 \leq k \leq 2 n} \Lambda_{k}^{D}(b, a)$.

Example 3.2 Suppose $a, b \in L^{1}[0,1], 0=t_{0}<t_{1}<\cdots<t_{2 n-1}<t_{2 n}=1, n \geq 1$, and

$$
\begin{aligned}
& a_{+} \cdot 1_{\left[t_{k}, t_{k+1}\right]} \succ 0, \quad b_{+} \cdot 1_{\left[t_{k}, t_{k+1}\right]}=0, \quad k=0,2,4, \ldots, 2 n-2, \\
& a_{+} \cdot 1_{\left[t_{k}, t_{k+1}\right]}=0, \quad b_{+} \cdot 1_{\left[t_{k}, t_{k+1}\right]} \succ 0, \quad k=1,3,5, \ldots, 2 n-1 .
\end{aligned}
$$

Then $\mathcal{H}_{p}^{D}(a, b) \cap \mathbb{R}^{+}=\bigcup_{1 \leq k \leq 2 n} \Lambda_{k}^{D}(a, b)$ and $\mathcal{H}_{p}^{D}(b, a) \cap \mathbb{R}^{+}=\bigcup_{1 \leq k \leq 2 n-1} \Lambda_{k}^{D}(b, a)$.

Example 3.3 Suppose $a, b \in L^{1}[0,1], 0=t_{0}<t_{1}<\cdots<t_{k}<t_{k+1}<1, \forall k \geq 1$, and

$$
\begin{array}{lll}
a_{+} \cdot 1_{\left[t_{k}, t_{k+1}\right]} \succ 0, & b_{+} \cdot 1_{\left[t_{k}, t_{k+1}\right]}=0, \quad \forall k=2 n, n \geq 0, \\
a_{+} \cdot 1_{\left[t_{k}, t_{k+1}\right]}=0, & b_{+} \cdot 1_{\left[t_{k}, t_{k+1}\right]} \succ 0, \quad \forall k=2 n-1, n \geq 1 .
\end{array}
$$

Then $\mathcal{H}_{p}^{D}(a, b) \cap \mathbb{R}^{+}=\bigcup_{1 \leq k \leq+\infty} \Lambda_{k}^{D}(a, b)$ and $\mathcal{H}_{p}^{D}(b, a) \cap \mathbb{R}^{+}=\bigcup_{1 \leq k \leq+\infty} \Lambda_{k}^{D}(b, a)$.

The following property can be proved by similar arguments as used for Property 3.1.

Property 3.2 Given $a, b \in L^{1}[0,1]$, we have the following results:

(i) if $a \prec 0$, then any positive half-eigenvalues $\Lambda_{k}^{N}(a, b), k \geq 0$, does not exist, namely

$$
\mathcal{H}_{p}^{N}(a, b) \cap \mathbb{R}^{+}=\emptyset
$$

(ii) if $a_{+} b_{+} \succ 0$, then those positive half-eigenvalues $\Lambda_{k}^{N}(a, b), k \geq 1$, exist, but the existence of a positive principal half-eigenvalue $\Lambda_{0}^{N}(a, b)=\lambda_{0}^{N}(a)$ is indefinite;

(iii) $\Lambda_{k+1}^{N}(a, b)$ exists, $k \geq 1 \Rightarrow$ both $\Lambda_{k}^{N}(a, b)$ and $\Lambda_{k}^{N}(b, a)$ exist;

(iv) both $\Lambda_{k}^{N}(a, b)$ and $\Lambda_{k}^{N}(b, a)$ exist, $k \geq 1 \Longrightarrow \Lambda_{k+1}^{N}(a, b)$ exists or $\Lambda_{k+1}^{N}(b, a)$ exists.

\subsection{Structure of the Fučík spectrum $\Pi_{p}^{D}(a, b)$}

In this subsection, we always use the notation

$$
\Theta(\lambda, \mu)=\Theta\left(-\frac{\pi_{p}}{2}, \lambda a, \mu b\right)
$$

for simplicity if there is no confusion. By Theorem 2.1, we see that $\Theta(\lambda, \mu)$ is continuous in $(\lambda, \mu) \in \mathbb{R}^{2}$. 
The following lemma tells us that $\Theta(\lambda, \mu)$ is quasi-monotonic in $\lambda$ and in $\mu$. This property is crucial for us to characterize the structure of the Fućík spectra $\Pi_{p}^{D}(a, b)$.

Lemma 3.7 Let $a, b \in L^{1}[0,1]$. The following results hold:

(i) if $\lambda^{*}>0, \mu^{*}>0$ and $\left(\lambda^{*}, \mu^{*}\right) \in W_{k}^{D}(a, b), k \geq 1$, then

$$
\Theta\left(\lambda, \mu^{*}\right) \begin{cases}>k \pi_{p}-\frac{\pi_{p}}{2}, & \text { if } \lambda \in\left(\lambda^{*},+\infty\right), \\ <k \pi_{p}-\frac{\pi_{p}}{2}, & \text { if } \lambda \in\left(0, \lambda^{*}\right) ;\end{cases}
$$

(ii) if $\lambda^{*}>0, \mu^{*}>0$ and $\left(\lambda^{*}, \mu^{*}\right) \in W_{k}^{D}(a, b), k \geq 2$, then

$$
\Theta\left(\lambda^{*}, \mu\right) \begin{cases}>k \pi_{p}-\frac{\pi_{p}}{2}, & \text { if } \mu \in\left(\mu^{*},+\infty\right) \\ <k \pi_{p}-\frac{\pi_{p}}{2}, & \text { if } \mu \in\left(0, \mu^{*}\right)\end{cases}
$$

Proof We only prove (i), and (ii) can be proved by similar arguments.

Suppose $\lambda^{*}>0, \mu^{*}>0$ and $\left(\lambda^{*}, \mu^{*}\right) \in W_{k}^{D}(a, b), k \geq 1$. It follows from the definition of $W_{k}^{D}(a, b)$ in equation (3.7) that

$$
\Theta\left(\lambda^{*}, \mu^{*}\right)=\Theta\left(-\frac{\pi_{p}}{2}, \lambda^{*} a, \mu^{*} b\right)=-\frac{\pi_{p}}{2}+k \pi_{p} .
$$

In the following, we write

$$
\theta(t)=\theta\left(t ; 0,-\frac{\pi_{p}}{2}, \lambda^{*} a, \mu^{*} b\right)
$$

for simplicity. By Lemma 2.2, there exist $(0=) t_{0}<t_{1}<t_{2}<\cdots<t_{k}(=1)$, such that

$$
\theta\left(t_{i}\right)=i \pi_{p}-\frac{\pi_{p}}{2}, \quad i=0,1,2, \ldots, k
$$

Furthermore, we can deduce that

$$
\begin{aligned}
& \theta(t)=\vartheta\left(t ; t_{2 i}, 2 i \pi_{p}-\frac{\pi_{p}}{2}, \lambda^{*} a\right), \quad \forall t \in\left[t_{2 i}, t_{2 i+1}\right], i=0,1, \ldots\left\lfloor\left\lfloor\frac{k-1}{2}\right\rfloor,\right. \\
& \theta(t)=\vartheta\left(t ; t_{2 i+1}, 2 i \pi_{p}+\frac{\pi_{p}}{2}, \mu^{*} b\right), \quad \forall t \in\left[t_{2 i+1}, t_{2 i+2}\right], i=0,1, \ldots,\left\lfloor\frac{k}{2}\right\rfloor-1 .
\end{aligned}
$$

Particularly, we get

$$
\begin{aligned}
& \vartheta\left(t_{2 i+1} ; t_{2 i}, 2 i \pi_{p}-\frac{\pi_{p}}{2}, \lambda^{*} a\right)=(2 i+1) \pi_{p}-\frac{\pi_{p}}{2}, \quad i=0,1, \ldots,\left\lfloor\frac{k-1}{2}\right\rfloor, \\
& \vartheta\left(t_{2 i+2} ; t_{2 i+1}, 2 i \pi_{p}+\frac{\pi_{p}}{2}, \mu^{*} b\right)=(2 i+1) \pi_{p}+\frac{\pi_{p}}{2}, \quad i=0,1, \ldots,\left\lfloor\frac{k}{2}\right\rfloor-1 .
\end{aligned}
$$

Then it follows from Lemma 3.6 that, for any $\lambda>\lambda^{*}(>0)$, we have

$$
\Theta\left(\lambda, \mu^{*}\right)=\theta\left(1 ; 0,-\frac{\pi_{p}}{2}, \lambda a, \mu^{*} b\right)>k \pi_{p}-\frac{\pi_{p}}{2} .
$$


To complete the proof of (i), we need only to prove

$$
\Theta\left(\lambda, \mu^{*}\right)<k \pi_{p}-\frac{\pi_{p}}{2}, \quad \forall \lambda \in\left(0, \lambda^{*}\right) .
$$

If this is not true, then there exists $\tilde{\lambda} \in\left(0, \lambda^{*}\right)$ such that $\Theta\left(\widetilde{\lambda}, \mu^{*}\right) \geq k \pi_{p}-\frac{\pi_{p}}{2}$. Write

$$
\tilde{\theta}(t)=\tilde{\theta}\left(t ; 0,-\frac{\pi_{p}}{2}, \tilde{\lambda} a, \mu^{*} b\right)
$$

for simplicity. Then there exist $(0=) s_{0}<s_{1}<s_{2}<\cdots<s_{k}(\leq 1)$ such that

$$
\widetilde{\theta}\left(s_{i}\right)=i \pi_{p}-\frac{\pi_{p}}{2}, \quad i=0,1,2, \ldots, k
$$

Since $\lambda^{*}>\tilde{\lambda}>0$, we can use similar arguments as in the previous paragraph to get

$$
\theta\left(s_{k} ; 0,-\frac{\pi_{p}}{2}, \lambda^{*} a, \mu^{*} b\right)>k \pi_{p}-\frac{\pi_{p}}{2} .
$$

Finally, it follows from the condition $s_{k} \leq 1$ and Lemma 2.2 that

$$
\Theta\left(\lambda^{*}, \mu^{*}\right)=\theta\left(1 ; 0,-\frac{\pi_{p}}{2}, \lambda^{*} a, \mu^{*} b\right)>k \pi_{p}-\frac{\pi_{p}}{2},
$$

a contradiction to the fact $\left(\lambda^{*}, \mu^{*}\right) \in W_{k}^{D}(a, b)$.

By the definition of $W_{1}^{D}(a, b)$ in equation (3.7), one has

$$
(\lambda, \mu) \in W_{1}^{D}(a, b) \Longleftrightarrow \theta\left(1 ; 0,-\frac{\pi_{p}}{2}, \lambda a, \mu b\right)=\frac{\pi_{p}}{2} .
$$

By the quasi-monotonicity results in Lemma 2.2, one has

$$
\theta\left(1 ; 0,-\frac{\pi_{p}}{2}, \lambda a, \mu b\right)=\frac{\pi_{p}}{2} \Longleftrightarrow \theta\left(1 ; 0,-\frac{\pi_{p}}{2}, \lambda a\right)=\frac{\pi_{p}}{2}
$$

Then

$$
(\lambda, \mu) \in W_{1}^{D}(a, b) \Longleftrightarrow \theta\left(1 ; 0,-\frac{\pi_{p}}{2}, \lambda a\right)=\frac{\pi_{p}}{2} .
$$

It follows from Lemma 3.1 that the solutions to

$$
\theta\left(1 ; 0,-\frac{\pi_{p}}{2}, \lambda a\right)=\frac{\pi_{p}}{2}
$$

are $\lambda=\lambda_{ \pm 1}^{D}(a)$ if $a_{ \pm} \succ 0, \lambda=\lambda_{1}^{D}(a)$ if $a \succ 0$, and $\lambda=\lambda_{-1}^{D}(a)$ if $a \prec 0$. Thus $W_{1}^{D}(a, b)$ can be characterized as in the following theorem.

Theorem 3.2 Let $a, b \in L^{1}[0,1]$ and assume that $a \neq 0$.

(i) If $a_{+} \succ 0$ and $a_{-} \succ 0$, then $W_{1}^{D}(a, b)=\left(\lambda_{1}^{D}(a) \times \mathbb{R}\right) \cup\left(\lambda_{-1}^{D}(a) \times \mathbb{R}\right)$. 
(ii) If $a \succ 0$, then $W_{1}^{D}(a, b)=\lambda_{1}^{D}(a) \times \mathbb{R}$.

(iii) If $a \prec 0$, then $W_{1}^{D}(a, b)=\lambda_{-1}^{D}(a) \times \mathbb{R}$.

Remark 3.1 It follows from equation (3.9) and the above theorem that $\widetilde{W}_{1}^{D}(a, b)$ is made up of two horizontal lines $\mathbb{R} \times \lambda_{1}^{D}(b)$ and $\mathbb{R} \times \lambda_{-1}^{D}(b)$. If the eigenvalues $\lambda_{1}^{D}(b)$ and $\lambda_{-1}^{D}(b)$ do not exist, then $\mathbb{R} \times \lambda_{1}^{D}(b)$ and $\mathbb{R} \times \lambda_{-1}^{D}(b)$ should be understood as empty sets, respectively.

If $k \geq 2$, the set $W_{k}^{D}(a, b)$ is more complicated than $W_{1}^{D}(a, b)$. As analyzed in Section 3.1, essentially we need only to discuss the subset $\Gamma_{k}^{D}(a, b)$ as defined in equation (3.13). Finally we will show that $\Gamma_{k}^{D}(a, b)$ is either an empty set, or a hyperbolic like curve. The following property helps us to locate $\Gamma_{k}^{D}(a, b)$ roughly.

Property 3.3 (i) $\left(\lambda^{*}, \mu^{*}\right) \in \Gamma_{2 k}^{D}(a, b), k \geq 1 \Longrightarrow \lambda^{*}>\lambda_{k}(a), \mu^{*}>\lambda_{k}(b)$.

(ii) $\left(\lambda^{*}, \mu^{*}\right) \in \Gamma_{2 k+1}^{D}(a, b), k \geq 1 \Longrightarrow \lambda^{*}>\lambda_{k+1}(a), \mu^{*}>\lambda_{k}(b)$.

Proof We will only prove (i), and (ii) can be proved similarly.

If $\left(\lambda^{*}, \mu^{*}\right) \in \Gamma_{2 k}^{D}(a, b)$, then $\lambda^{*} \geq 0, \mu^{*} \geq 0$, and there exist $0=t_{0}<t_{1}<\cdots<t_{2 k-1}<t_{2 k}=1$, such that

$$
\theta(t):=\theta\left(t ; 0,-\frac{\pi_{p}}{2}, \lambda^{*} a, \mu^{*} b\right)
$$

satisfies

$$
\theta\left(t_{i}\right)=i \pi_{p}-\frac{\pi_{p}}{2}, \quad i=0,1,2, \ldots, 2 k .
$$

Therefore $\theta(t)$ satisfies equation (3.45) on each interval $I_{i}=\left[t_{2 i-2}, t_{2 i-1}\right], i=1,2, \ldots, k$, and it satisfies equation (3.49) on each interval $J_{i}=\left[t_{2 i-1}, t_{2 i}\right], i=1,2, \ldots, k$. In other words, we have

$$
\begin{array}{ll}
\theta(t)=\vartheta\left(t ; t_{2 i-2},(2 i-2) \pi_{p}-\frac{\pi_{p}}{2}, \lambda^{*} a\right), & t \in I_{i}, i=1,2, \ldots, k \\
\theta(t)=\vartheta\left(t ; t_{2 i-1},(2 i-1) \pi_{p}-\frac{\pi_{p}}{2}, \mu^{*} b\right), & t \in J_{i}, i=1,2, \ldots, k .
\end{array}
$$

Take the following notations for simplicity:

$$
\begin{aligned}
& \vartheta(t)=\vartheta\left(t ; 0,-\frac{\pi_{p}}{2}, \lambda^{*} a\right), \quad \forall t \in[0,1], \\
& \widetilde{\vartheta}(t)=\vartheta\left(t ; 0,-\frac{\pi_{p}}{2}, \mu^{*} b\right), \quad \forall t \in[0,1] .
\end{aligned}
$$

Let $i=1$ and take $t=t_{1}$ in equation (3.62). Then it follows from equation (3.61) that

$$
\vartheta\left(t_{1}\right)=\theta\left(t_{1}\right)=\frac{\pi_{p}}{2} .
$$

By the quasi-monotonicity results in Lemma 2.2, we have

$$
\vartheta\left(t_{2}\right)=\vartheta\left(t_{2} ; t_{1}, \vartheta\left(t_{1}\right), \lambda^{*} a\right)=\vartheta\left(t_{2} ; t_{1}, \frac{\pi_{p}}{2}, \lambda^{*} a\right)>\frac{\pi_{p}}{2},
$$


and hence

$$
\begin{aligned}
\vartheta\left(t_{3}\right) & =\vartheta\left(t_{3} ; t_{2}, \vartheta\left(t_{2}\right), \lambda^{*} a\right) \\
& >\vartheta\left(t_{3} ; t_{2}, \frac{\pi_{p}}{2}, \lambda^{*} a\right) \quad(\text { by equation }(3.64)) \\
& \left.=\vartheta\left(t_{3} ; t_{2}, \frac{3 \pi_{p}}{2}, \lambda^{*} a\right)-\pi_{p} \quad \text { (by equation }(3.16)\right) \\
& =\theta\left(t_{3}\right)-\pi_{p} \quad(\text { by equation }(3.62)) \\
& =\frac{3 \pi_{p}}{2} \quad(\text { by equation }(3.61)) .
\end{aligned}
$$

Inductively, we can show that

$$
\vartheta\left(t_{2 i-1}\right)>i \pi_{p}-\frac{\pi_{p}}{2}, \quad \vartheta\left(t_{2 i}\right)>i \pi_{p}-\frac{\pi_{p}}{2}, \quad i=2,3, \ldots, k .
$$

Similarly, applying equations (3.16), (3.61), (3.63), and Lemma 2.2, we can obtain

$$
\widetilde{\vartheta}\left(t_{2 i-1}\right)>i \pi_{p}-\frac{3 \pi_{p}}{2}, \quad \widetilde{\vartheta}\left(t_{2 i}\right)>i \pi_{p}-\frac{\pi_{p}}{2}, \quad i=1,2, \ldots, k
$$

The case $i=k(\geq 1)$ in equation (3.65) implies that

$$
\vartheta\left(1 ; 0,-\frac{\pi_{p}}{2}, \lambda^{*} a\right)>k \pi_{p}-\frac{\pi_{p}}{2} .
$$

By equation (3.36), we see that $\lambda^{*} \neq 0$, and hence $\lambda^{*}>0$. By the definition of $\lambda_{k}^{D}(a)$ as in equation (3.26), one has

$$
\vartheta\left(1 ; 0,-\frac{\pi_{p}}{2}, \lambda_{k}^{D}(a) a\right)=k \pi_{p}-\frac{\pi_{p}}{2} .
$$

Then it follows from Lemma 3.4(i) that $\lambda^{*}>\lambda_{k}^{D}(a)(>0)$.

Similarly, it can be deduced from equations (3.66), (3.26) and Lemma 3.4 that $\mu^{*}>$ $\lambda_{k}^{D}(b)$.

Theorem 3.3 If $\Gamma_{k}^{D}(a, b) \neq \emptyset, k \geq 2$, then $\Gamma_{k}^{D}(a, b)$ is a continuous, strictly decreasing, hyperbolic like curve

$$
\begin{aligned}
& f_{k}:\left(\alpha_{k},+\infty\right) \longmapsto\left(\beta_{k},+\infty\right), \\
& \lambda \longmapsto \mu=f_{k}(\lambda),
\end{aligned}
$$

with the horizontal asymptotic line $\mu=\beta_{k}$ and the vertical asymptotic line $\lambda=\alpha_{k}$, where $\alpha_{k} \geq \lambda_{\left\lfloor\frac{k+1}{2}\right\rfloor}^{D}(a), \beta_{k} \geq \lambda_{\left\lfloor\frac{k}{2}\right\rfloor}^{D}(b)$, and $\lfloor\cdot\rfloor$ is the floor function.

Proof Assume that $\Gamma_{k}^{D}(a, b) \neq \emptyset, k \geq 2$. We prove the theorem by seven steps.

Step 1. We aim to prove that $\Gamma_{k}^{D}(a, b)$ is not a single-point set. Suppose that $\left(\lambda^{*}, \mu^{*}\right) \in$ $\Gamma_{k}^{D}(a, b)$, then

$$
\Theta\left(\lambda^{*}, \mu^{*}\right)=\Theta\left(-\frac{\pi_{p}}{2}, \lambda^{*} a, \mu^{*} b\right)=k \pi_{p}-\frac{\pi_{p}}{2} .
$$


By Property 3.3, $\lambda^{*}>\lambda_{\left\lfloor\frac{k+1}{2}\right\rfloor}^{D}(a)>0$ and $\mu^{*}>\lambda_{\left\lfloor\frac{k}{2}\right\rfloor}^{D}(b)>0$. Then it follows from Lemma 3.7 that

$$
\Theta\left(\frac{\lambda^{*}}{2}, \mu^{*}\right)<k \pi_{p}-\frac{\pi_{p}}{2}<\Theta\left(\lambda^{*}, 2 \mu^{*}\right) .
$$

Since $\Theta(\lambda, \mu)$ is continuous in $(\lambda, \mu) \in \mathbb{R}^{2}$, there must exist a point $(\tilde{\lambda}, \tilde{\mu})$ in the open line segment with endpoints $\left(\frac{\lambda^{*}}{2}, \mu^{*}\right)$ and $\left(\lambda^{*}, 2 \mu^{*}\right)$, and hence $\frac{\lambda^{*}}{2}<\tilde{\lambda}<\lambda^{*}$ and $\mu^{*}<\tilde{\mu}<2 \mu^{*}$, such that

$$
\Theta(\widetilde{\lambda}, \tilde{\mu})=k \pi_{p}-\frac{\pi_{p}}{2}
$$

Thus $(\tilde{\lambda}, \tilde{\mu}) \neq\left(\lambda^{*}, \mu^{*}\right)$ and $(\tilde{\lambda}, \tilde{\mu}) \in \Gamma_{k}^{D}(a, b)$, proving the claim. Furthermore, let

$$
A_{k}:=\left\{\lambda \in \mathbb{R}: \exists \mu \in \mathbb{R} \text {, s.t. }(\lambda, \mu) \in \Gamma_{k}^{D}(a, b)\right\} \subset\left(\lambda_{\left\lfloor\frac{k+1}{2}\right\rfloor}^{D}(b),+\infty\right),
$$

then $A_{k} \neq \emptyset$ is not a single-point set, because $\tilde{\lambda} \in A_{k}, \lambda^{*} \in A_{k}$ and $\tilde{\lambda} \neq \lambda^{*}$.

Step 2. It follows from Lemma 3.7 that every horizontal line intersects $\Gamma_{k}^{D}(a, b)$ at one time at most, so does every vertical line. Therefore $\Gamma_{k}^{D}(a, b)$ is the image of some function

$$
\mu=f_{k}(\lambda), \quad t \in A_{k} .
$$

Suppose $\lambda_{1}, \lambda_{2} \in A_{k}$ and $\lambda_{1}<\lambda_{2}$. Let $\mu_{i}=f_{k}\left(\lambda_{i}\right), i=1$, 2. If $\mu_{1} \leq \mu_{2}$, then it follows from Lemma 3.7 that

$$
k \pi_{p}-\frac{\pi_{p}}{2}=\Theta\left(\lambda_{2}, \mu_{2}\right) \geq \Theta\left(\lambda_{2}, \mu_{1}\right)>\Theta\left(\lambda_{1}, \mu_{1}\right)=k \pi_{p}-\frac{\pi_{p}}{2},
$$

which is a contradiction. Thus $\mu_{1}>\mu_{2}$. Therefore $f_{k}$ is a strictly decreasing function on $A_{k}$. Let

$$
B_{k}:=\left\{\mu=f_{k}(\lambda): \lambda \in A_{k}\right\}
$$

and

$$
\alpha_{k}=\inf \left\{\lambda: \lambda \in A_{k}\right\}, \quad \beta_{k}=\inf \left\{\mu: \mu \in B_{k}\right\}
$$

By Property 3.3, $\alpha_{k} \geq \lambda_{\left\lfloor\frac{k+1}{2}\right\rfloor}^{D}(a)>0$ and $\beta_{k} \geq \lambda_{\left\lfloor\frac{k}{2}\right\rfloor}^{D}(b)>0$.

Step 3. We claim that if $\lambda_{1}, \lambda_{2} \in A_{k}$ and $\lambda_{1}<\lambda_{2}$, then $\lambda^{*} \in A_{k}$ for any $\lambda^{*} \in\left(\lambda_{1}, \lambda_{2}\right)$. Let $\mu_{i}=$ $f_{k}\left(\lambda_{i}\right), i=1,2$. By the monotonicity result proved in Step 2 , we have $\mu_{1}>\mu_{2}$. By Lemma 3.7 again, for any $\lambda^{*} \in\left(\lambda_{1}, \lambda_{2}\right)$, one has

$$
\Theta\left(\lambda^{*}, \mu_{1}\right)>\Theta\left(\lambda_{1}, \mu_{1}\right)=k \pi_{p}-\frac{\pi_{p}}{2}=\Theta\left(\lambda_{2}, \mu_{2}\right)>\Theta\left(\lambda^{*}, \mu_{2}\right) .
$$

Then the continuity of $\Theta(\lambda, \mu)$ in $(\lambda, \mu) \in \mathbb{R}^{2}$ implies the existence of $\mu^{*} \in\left(\mu_{2}, \mu_{1}\right)$ such that

$$
\Theta\left(\lambda^{*}, \mu^{*}\right)=k \pi_{p}-\frac{\pi_{p}}{2}
$$

and hence $\left(\lambda^{*}, \mu^{*}\right) \in \Gamma_{k}^{D}(a, b)$, completing the proof of the claim. 
Step 4. We aim to prove that $A_{k}=\left(\alpha_{k},+\infty\right)$. If $\alpha_{k} \in A_{k}$, then $\left(\alpha_{k}, f_{k}\left(\alpha_{k}\right)\right) \in \Gamma_{k}^{D}(a, b)$. Thus

$$
\Theta\left(\alpha_{k}, f_{k}\left(\alpha_{k}\right)\right)=k \pi_{p}-\frac{\pi_{p}}{2},
$$

$\alpha_{k}>0$ and $f_{k}\left(\alpha_{k}\right)>0$. Similar arguments as in Step 1 show that there exists some point $\left(\lambda^{*}, \mu^{*}\right)$ in the open line segment with endpoints $\left(\frac{1}{2} \alpha_{k}, f_{k}\left(\alpha_{k}\right)\right)$ and $\left(\alpha_{k}, 2 f_{k}\left(\alpha_{k}\right)\right)$, such that $\left(\lambda^{*}, \mu^{*}\right) \in \Gamma_{k}^{D}(a, b)$, and hence $\lambda^{*}<\alpha_{k}=\inf \left\{\lambda: \lambda \in A_{k}\right\}$ and $\lambda^{*} \in A_{k}$, a contradiction. Therefore $a_{k} \notin A_{k}$. Similarly, we can prove that $\sup \left\{\lambda: \lambda \in A_{k}\right\}=+\infty$. Combining the results in Step 3, we see that $A_{k}=\left(\alpha_{k},+\infty\right)$.

Step 5. We aim to prove that $B_{k}=\left(\beta_{k},+\infty\right)$. Since $A_{k}=\left(\alpha_{k},+\infty\right)$ and the function $f_{k}$ : $A_{k} \rightarrow B_{k}$ is strictly decreasing, we see that $B_{k} \neq \emptyset$ is not a single-point set. Then similar arguments as in Step 3 and Step 4 show that $B_{k}=\left(\beta_{k},+\infty\right)$.

Step 6. We aim to show that $f_{k}$ is a continuous function on $A_{k}=\left(\alpha_{k},+\infty\right)$. In fact, we have

$$
\Theta\left(\lambda, f_{k}(\lambda)\right)=k \pi_{p}-\frac{\pi_{p}}{2}, \quad \forall \lambda \in\left(\alpha_{k},+\infty\right) .
$$

Fix any $\lambda^{*} \in\left(\alpha_{k},+\infty\right)$. By the monotonicity results proved in Step 2, we see that both

$$
\mu_{1}:=f_{k}\left(\lambda^{*}-\right)=\lim _{\lambda<\lambda^{*}, \lambda \rightarrow \lambda^{*}} f_{k}(\lambda) \quad \text { and } \quad \mu_{2}:=f_{k}\left(\lambda^{*}+\right)=\lim _{\lambda>\lambda^{*}, \lambda \rightarrow \lambda^{*}} f_{k}(\lambda)
$$

exist, and $\mu_{1} \geq f_{k}\left(\lambda^{*}\right) \geq \mu_{2}$. Furthermore, we can show that $\mu_{2} \geq f_{k}\left(\lambda^{*}+1\right)>0$. Thus $\mu_{1} \geq$ $\mu_{2}>0$. Let $\lambda<\lambda^{*}$ and $\lambda \rightarrow \lambda^{*}$ in equation (3.67), then the continuity of $\Theta(\lambda, \mu)$ in $(\lambda, \mu) \in$ $\mathbb{R}^{2}$ guarantees that

$$
\Theta\left(\lambda^{*}, \mu_{1}\right)=k \pi_{p}-\frac{\pi_{p}}{2} .
$$

Similarly, let $\lambda>\lambda^{*}$ and $\lambda \rightarrow \lambda^{*}$ in equation (3.67), then

$$
\Theta\left(\lambda^{*}, \mu_{2}\right)=k \pi_{p}-\frac{\pi_{p}}{2} .
$$

Now Lemma 3.7 implies that $\mu_{1}=\mu_{2}=f_{k}\left(\lambda^{*}\right)$. Thus $f_{k}$ is continuous at the point $\lambda^{*}$. Since $\lambda^{*} \in\left(\alpha_{k},+\infty\right)$ can be chosen arbitrarily, $f_{k}$ is continuous in $\lambda \in\left(\alpha_{k},+\infty\right)$.

Step 7. In the above six steps, we have shown that the continuous and strictly decreasing function $\mu=f_{k}(\lambda)$ maps $\left(\alpha_{k},+\infty\right)$ onto $\left(\beta_{k},+\infty\right)$. Then it is necessary that

$$
\lim _{\lambda>\alpha_{k}, \lambda \rightarrow \alpha_{k}} f_{k}(\lambda)=+\infty \text { and } \lim _{\lambda \rightarrow+\infty} f_{k}(\lambda)=\beta_{k} .
$$

Therefore $\mu=f_{k}(\lambda)$ is a hyperbolic like curve with the horizontal asymptotic line $\mu=\beta_{k}$ and the vertical asymptotic line $\lambda=\alpha_{k}$.

When the weight $a$ or $b$ is positive, we can improve the results about the asymptotic lines in Theorem 3.3.

Theorem 3.4 Let $a, b \in L^{1}[0,1]$. The following results hold.

(i) If $a_{+}>0$ and $b>0$, then $\Gamma_{k}^{D}(a, b) \neq \emptyset$ for any $k \geq 2$, and the vertical asymptotic line of $\Gamma_{k}^{D}(a, b)$ is $\lambda=\lambda_{\left\lfloor\frac{k+1}{2}\right\rfloor}^{D}(a)$. 
(ii) If $a \succ 0$ and $b_{+}>0$, then $\Gamma_{k}^{D}(a, b) \neq \emptyset$ for any $k \geq 2$, and the horizontal asymptotic line of $\Gamma_{k}^{D}(a, b)$ is $\mu=\lambda_{\left\lfloor\frac{k}{2}\right\rfloor}^{D}(b)$.

Proof We only prove (i) for the case $k=2 n, n \geq 1$. Other cases can be proved similarly.

Since $a_{+}>0$, it follows from Lemma 3.1 that the eigenvalue $\lambda_{n}^{D}(a)$ exists. By the definition of $\lambda_{n}^{D}(a)$ as in equation (3.26), we have $\vartheta\left(1 ; 0,-\frac{\pi_{p}}{2}, \lambda_{n}^{D}(a) a\right)=n \pi_{p}-\frac{\pi_{p}}{2}$. By Lemma 2.2, there exist $0=t_{0}<t_{1} \cdots<t_{n-1}<t_{n}=1$ such that

$$
\vartheta\left(t_{i} ; 0,-\frac{\pi_{p}}{2}, \lambda_{n}^{D}(a) a\right)=i \pi_{p}-\frac{\pi_{p}}{2}, \quad i=0,1,2, \ldots, n,
$$

and hence

$$
\vartheta\left(t_{i+1} ; t_{i}, i \pi_{p}-\frac{\pi_{p}}{2}, \lambda_{n}^{D}(a) a\right)=i \pi_{p}+\frac{\pi_{p}}{2}, \quad i=0,1,2, \ldots, n-1 .
$$

By equation (3.16), we also have

$$
\vartheta\left(t_{i+1} ; t_{i}, 2 i \pi_{p}-\frac{\pi_{p}}{2}, \lambda_{n}^{D}(a) a\right)=2 i \pi_{p}+\frac{\pi_{p}}{2}, \quad i=0,1,2, \ldots, n-1 .
$$

Given any $\lambda^{*}>\lambda_{n}^{D}(a)$, it follows from Lemma 3.4(i) that

$$
\vartheta\left(t_{i+1} ; t_{i}, 2 i \pi_{p}-\frac{\pi_{p}}{2}, \lambda^{*} a\right)>2 i \pi_{p}+\frac{\pi_{p}}{2}
$$

and hence there exist $s_{i} \in\left(t_{i}, t_{i+1}\right)$, such that

$$
\vartheta\left(s_{i} ; t_{i}, 2 i \pi_{p}-\frac{\pi_{p}}{2}, \lambda^{*} a\right)=2 i \pi_{p}+\frac{\pi_{p}}{2}, \quad i=0,1,2, \ldots, n-1 .
$$

We have $b \cdot 1_{\left[s_{i}, t_{i+1}\right]} \succ 0$ because $b>0$. By Lemma 3.3, there exist $\mu_{i}>0$, such that

$$
\vartheta\left(t_{i+1} ; s_{i}, 2 i \pi_{p}+\frac{\pi_{p}}{2}, \mu_{i} b\right)=(2 i+1) \pi_{p}+\frac{\pi_{p}}{2}, \quad i=0,1,2, \ldots, n-1 .
$$

Define a weight $q$ on the interval $[0,1]$ as

$$
q(t)=\mu_{i} b(t), \quad \forall t \in\left[t_{i}, t_{i+1}\right], i=0,1,2, \ldots, n-1 .
$$

Then it follows from equations (3.68) and (3.69) that

$$
\theta\left(1 ; 0,-\frac{\pi_{p}}{2}, \lambda^{*} a, q\right)=2 n \pi_{p}-\frac{\pi_{p}}{2} .
$$

Let $\bar{\mu}:=\max _{0 \leq i \leq n-1}\left\{\mu_{i}\right\}$ and $\underline{\mu}:=\min _{0 \leq i \leq n-1}\left\{\mu_{i}\right\}$. One has $\bar{\mu} b \geq q \geq \underline{\mu} b$ on $[0,1]$. Now Remark 2.2 implies that

$$
\begin{aligned}
& \Theta\left(\lambda^{*}, \bar{\mu}\right)=\theta\left(1 ; 0,-\frac{\pi_{p}}{2}, \lambda^{*} a, \bar{\mu} b\right) \geq \theta\left(1 ; 0,-\frac{\pi_{p}}{2}, \lambda^{*} a, q\right)=2 n \pi_{p}-\frac{\pi_{p}}{2} \\
& \Theta\left(\lambda^{*}, \underline{\mu}\right)=\theta\left(1 ; 0,-\frac{\pi_{p}}{2}, \lambda^{*} a, \underline{\mu b}\right) \leq \theta\left(1 ; 0,-\frac{\pi_{p}}{2}, \lambda^{*} a, q\right)=2 n \pi_{p}-\frac{\pi_{p}}{2} .
\end{aligned}
$$


By the continuity of $\Theta(\lambda, \mu)$ in $(\lambda, \mu)$, there exists $\mu^{*} \in[\mu, \bar{\mu}]$, and hence $\mu^{*}>0$, such that $\Theta\left(\lambda^{*}, \mu^{*}\right)=2 n \pi_{p}-\frac{\pi_{p}}{2}$. Thus $\left(\lambda^{*}, \mu^{*}\right) \in \Gamma_{2 n}^{D}(a, b)$ and $\left.\Gamma_{2 n}^{D} \overline{(a}, b\right) \neq \emptyset$.

Now it follows from Theorem 3.3 that $\Gamma_{2 n}^{D}(a, b)$ is a hyperbolic like curve and its vertical asymptotic line $\lambda=\alpha_{2 n}$ satisfies $\alpha_{2 n} \geq \lambda_{n}^{D}(a)$. On the other hand, since $\left(\lambda^{*}, \mu^{*}\right) \in \Gamma_{2 n}^{D}(a, b)$, we get $\alpha_{2 n}<\lambda^{*}$. Furthermore, $\alpha_{2 n} \leq \lambda_{n}^{D}(a)$ because $\lambda^{*}>\lambda_{n}^{D}(a)$ can be chosen arbitrarily. Therefore the vertical asymptotic line of $\Gamma_{2 n}^{D}(a, b)$ is $\lambda=\lambda_{n}^{D}(a)$.

If $\lambda^{*}=\Lambda_{k}^{D}(a, b)$ exists and $k \geq 2$, then it follows from equation (3.37) that

$$
\Theta\left(\lambda^{*}, \lambda^{*}\right)=k \pi_{p}-\frac{\pi_{p}}{2}
$$

and hence $\left(\lambda^{*}, \lambda^{*}\right) \in \Gamma_{k}^{D}(a, b)$. Thus $\Gamma_{k}^{D}(a, b)$ is a hyperbolic like curve by Theorem 3.3. On the other hand, suppose that $\Gamma_{k}^{D}(a, b), k \geq 2$, is a hyperbolic like curve. Theorem 3.3 tells us it has a horizontal asymptotic line and a vertical one. Then it must intersect the diagonal $\mu=\lambda$ at a unique point $\left(\lambda_{*}, \lambda_{*}\right)$. Furthermore, we can deduce that $\lambda_{*}=\Lambda_{k}^{D}(a, b)$. In conclusion, we have the following property.

Property 3.4 Let $a, b \in L^{1}[0,1]$ and $k \geq 2$. The following results hold.

(i) $\Gamma_{k}^{D}(a, b)=\emptyset$ if and only if $\Lambda_{k}^{D}(a, b)$ does not exist.

(ii) $\Gamma_{k}^{D}(a, b)$ is a hyperbolic like curve if and only if $\Lambda_{k}^{D}(a, b)$ exists.

(iii) $\Gamma_{k}^{D}(a, b)$ is either an empty set or a hyperbolic like curve in the quadrant $\mathbb{R}^{+} \times \mathbb{R}^{+}$ emanating from the point $\left(\Lambda_{k}^{D}(a, b), \Lambda_{k}^{D}(a, b)\right)$.

Remark 3.2 If $\Gamma_{k+1}^{D}(a, b) \neq \emptyset, k \geq 2$, then it follows from Properties 3.1 and 3.4 that $\Gamma_{k}^{D}(a, b) \neq \emptyset$. Furthermore, we can deduce by Lemma 3.7 that the higher-order hyperbolic like curve $\Gamma_{k+1}^{D}(a, b)$ always lies above the lower-order curve $\Gamma_{k}^{D}(a, b)$.

For any $k \geq 2$, denote $\mathbb{R}_{+}=[0,+\infty)$ and define

$$
\widetilde{\Gamma}_{k}^{D}(a, b):=\widetilde{W}_{k}^{D}(a, b) \cap\left(\mathbb{R}_{+} \times \mathbb{R}_{+}\right) .
$$

By equations (3.9) and (3.13), we know that $\widetilde{\Gamma}_{k}^{D}(a, b)$ is asymmetric to $\Gamma_{k}^{D}(b, a)$ about the diagonal $\mu=\lambda$. Namely,

$$
(\lambda, \mu) \in \widetilde{\Gamma}_{k}^{D}(a, b) \Longleftrightarrow(\mu, \lambda) \in \Gamma_{k}^{D}(b, a) .
$$

Then we get can the following results immediately from Property 3.4.

Property 3.5 Let $a, b \in L^{1}[0,1]$ and $k \geq 2$. Then $\widetilde{\Gamma}_{k}^{D}(a, b)$ is either an empty set or a hyperbolic like curve in the quadrant $\mathbb{R}^{+} \times \mathbb{R}^{+}$emanating from $\left(\Lambda_{k}^{D}(b, a), \Lambda_{k}^{D}(b, a)\right)$, and $\widetilde{\Gamma}_{k}^{D}(a, b) \neq \emptyset$ if and only if $\Lambda_{k}^{D}(b, a)$ exists.

By Properties 3.4 and 3.5, we see that the existence of those hyperbolic like curves $\Gamma_{k}^{D}(a, b)$ and $\widetilde{\Gamma}_{k}^{D}(a, b), k \geq 2$, is determined by the existence of those half-eigenvalues $\Lambda_{k}^{D}(a, b)$ and $\Lambda_{k}^{D}(b, a)$, respectively. By Corollary 3.1, we can conclude that besides those trivial lines, the Fučík spectrum $\Pi_{p}^{D}(a, b)$ confined to the quadrant $\mathbb{R}^{+} \times \mathbb{R}^{+}$is an empty 
set, or made up of an odd number of hyperbolic like curves, or made up of a double sequence of hyperbolic like curves. Taking the relations (3.10)-(3.12) into consideration, we obtain the following theorem.

Theorem 3.5 Let $a, b \in L^{1}[0,1]$. Then

$$
\begin{aligned}
& \Pi_{p}^{D}(a, b) \supset W_{1}^{D}(a, b)=\left(\lambda_{1}^{D}(a) \times \mathbb{R}\right) \cup\left(\lambda_{-1}^{D}(a) \times \mathbb{R}\right), \\
& \Pi_{p}^{D}(a, b) \supset \widetilde{W}_{1}^{D}(a, b)=\left(\mathbb{R} \times \lambda_{1}^{D}(b)\right) \cup\left(\mathbb{R} \times \lambda_{-1}^{D}(b)\right) .
\end{aligned}
$$

If one of the half-eigenvalues $\lambda_{ \pm 1}^{D}(a)$ or $\lambda_{ \pm 1}^{D}(b)$ does not exist, the corresponding straight line $\lambda_{ \pm 1}^{D}(a) \times \mathbb{R}$ or $\mathbb{R} \times \lambda_{ \pm 1}^{D}(b)$ should be understood as an empty set. Let

$$
\check{\Pi}_{p}^{D}(a, b):=\Pi_{p}^{D}(a, b) \backslash\left(W_{1}^{D}(a, b) \cup \widetilde{W}_{1}^{D}(a, b)\right),
$$

then $\check{\Pi}_{p}^{D}(a, b) \cap\left(\mathbb{R}^{+} \times \mathbb{R}^{+}\right)$consists of zero, an odd number of, or a double sequence of hyperbolic like curves:

$$
\check{\Pi}_{p}^{D}(a, b) \cap\left(\mathbb{R}^{+} \times \mathbb{R}^{+}\right)=\bigcup_{k \in \mathbb{Z}, k \geq 2}\left(\Gamma_{k}^{D}(a, b) \cup \widetilde{\Gamma}_{k}^{D}(a, b)\right) .
$$

If $\Lambda_{k}^{D}(a, b)$ or $\Lambda_{k}^{D}(b, a)$ does not exist, $\Gamma_{k}^{D}(a, b)$ or $\widetilde{\Gamma}_{k}^{D}(a, b)$ should be understood as an empty set, respectively. If $\Lambda_{k}^{D}(a, b)$ and $\Lambda_{k}^{D}(b, a)$ exist, $\Gamma_{k}^{D}(a, b)$ and $\widetilde{\Gamma}_{k}^{D}(a, b)$ are continuous, strictly decreasing, hyperbolic like curves. Moreover,

(i) $\check{\Pi}_{p}^{D}(a, b) \cap\left(\mathbb{R}^{-} \times \mathbb{R}^{+}\right)$is asymmetric to $\check{\Pi}_{p}^{D}(-a, b) \cap\left(\mathbb{R}^{+} \times \mathbb{R}^{+}\right)$about the vertical line $\lambda=0$;

(ii) $\check{\Pi}_{p}^{D}(a, b) \cap\left(\mathbb{R}^{-} \times \mathbb{R}^{-}\right)$is asymmetric to $\check{\Pi}_{p}^{D}(-a,-b) \cap\left(\mathbb{R}^{+} \times \mathbb{R}^{+}\right)$about the origin $(\lambda, \mu)=(0,0)$;

(iii) $\check{\Pi}_{p}^{D}(a, b) \cap\left(\mathbb{R}^{+} \times \mathbb{R}^{-}\right)$is asymmetric to $\check{\Pi}_{p}^{D}(a,-b) \cap\left(\mathbb{R}^{+} \times \mathbb{R}^{+}\right)$about the horizontal line $\mu=0$.

\subsection{Fučík spectrum $\Pi_{p}^{D}(a, b)$ with positive weights}

Assume that $a, b \in L^{1}[0,1], a>0$ and $b>0$. Then it follows from Lemma 3.1 that $\lambda_{1}^{D}(a)$ and $\lambda_{1}^{D}(b)$ exist, but $\lambda_{-1}^{D}(a)$ and $\lambda_{-1}^{D}(b)$ do not exist. By Property 3.1 and Example 3.1, all halfeigenvalues $\Lambda_{k}^{D}(a, b)$ and $\Lambda_{k}^{D}(b, a), k \geq 2$, exist; but none of the half-eigenvalues $\Lambda_{k}^{D}(-a, b)$, $\Lambda_{k}^{D}(b,-a), \Lambda_{k}^{D}(-a,-b), \Lambda_{k}^{D}(-b,-a), \Lambda_{k}^{D}(a,-b), \Lambda_{k}^{D}(-b, a), k \geq 2$, exist. Then we have the following theorem.

Theorem 3.6 Let $a, b \in L^{1}[0,1], a>0$ and $b>0$. Then $\Pi_{p}^{D}(a, b)$ is made up of one vertical line, one horizontal line and a double sequence of differentiable, strictly decreasing, hyperbolic like curves in $\mathbb{R}^{+} \times \mathbb{R}^{+}$:

$$
\Pi_{p}^{D}(a, b)=\left(\lambda_{1}^{D}(a) \times \mathbb{R}\right) \cup\left(\mathbb{R} \times \lambda_{1}^{D}(b)\right) \bigcup_{k \geq 2}\left(\Gamma_{k}^{D}(a, b) \cup \widetilde{\Gamma}_{k}^{D}(a, b)\right)
$$

For each $k \geq 2, \Gamma_{k}^{D}(a, b)$ has the vertical asymptotic line $\lambda=\lambda_{\left\lfloor\frac{k+1}{2}\right\rfloor}^{D}(a)$ and the horizontal asymptotic line $\mu=\lambda_{\left\lfloor\frac{k}{2}\right\rfloor}^{D}(b)$. 
Proof We need only to prove the differentiability of $\Gamma_{k}^{D}(a, b)$. Recall from Theorem 3.3 that for any $k \geq 2$, the curve $\Gamma_{k}^{D}(a, b):\left(\alpha_{k},+\infty\right) \rightarrow\left(\beta_{k},+\infty\right), \mu=f_{k}(\lambda)$ is determined by

$$
\Theta(\lambda, \mu):=\Theta\left(-\frac{\pi_{p}}{2} ; \lambda a, \mu b\right)=k \pi_{p}-\frac{\pi_{p}}{2}, \quad \lambda>0, \mu>0 .
$$

Given $\left(\lambda^{*}, \mu^{*}\right) \in \Gamma_{k}^{D}(a, b), k \geq 2$, denote the associated eigenfunction by $X(t)$. Then $X_{-} \succ 0$ because $k \geq 2$. Since $b(t)>0$ for almost every $t \in[0,1]$, it follows from formulation (2.7) that

$$
\left.\frac{\partial}{\partial \mu} \Theta(\lambda, \mu)\right|_{\left(\lambda^{*}, \mu^{*}\right) \in \Gamma_{k}^{D}(a, b)}=\mu^{*} \int_{0}^{1} X_{-}^{p}(t) b(t) d t>0 .
$$

Thus the Implicit Function Theorem can be applied to equation (3.70), and we see that the hyperbolic like curve $\Gamma_{k}^{D}(a, b)$ is differentiable and

$$
f_{k}^{\prime}(\lambda)=-\frac{\frac{\partial}{\partial \lambda} \Theta(\lambda, \mu)}{\frac{\partial}{\partial \mu} \Theta(\lambda, \mu)} .
$$

\section{Fučík spectrum for Neumann problems}

Given a pair of indefinite weights $a, b \in L^{1}[0,1]$, the (Neumann type) Fučík spectrum $\Pi_{p}^{N}(a, b)$ is defined as the set of those $(\lambda, \mu) \in \mathbb{R}^{2}$ such that system (1.2)-(1.4) has nontrivial solutions.

Via similar arguments as in the previous sections, $\Pi_{p}^{N}(a, b)$ can also be characterized. We list the results in the following but omit the detailed proof.

Theorem 4.1 The Neumann Fuč́k spectrum $\Pi_{p}^{N}(a, b)$ can be decomposed as

$$
\Pi_{p}^{N}(a, b)=\bigcup_{k \in \mathbb{Z}, k \geq 0}\left(W_{k}^{N}(a, b) \cup \widetilde{W}_{k}^{N}(a, b)\right)
$$

where $W_{k}^{N}(a, b)$ and $\widetilde{W}_{k}^{N}(a, b)$ are defined as

$$
\begin{aligned}
& W_{k}^{N}(a, b)=\left\{(\lambda, \mu) \in \mathbb{R}^{2}: \theta(1 ; 0,0, \lambda a, \mu b)=k \pi_{p}\right\}, \\
& \widetilde{W}_{k}^{N}(a, b)=W_{k}^{N}(b, a)=\left\{(\lambda, \mu) \in \mathbb{R}^{2}: \theta(1 ; 0,0, \mu b, \lambda a)=k \pi_{p}\right\},
\end{aligned}
$$

and hence

$$
(\lambda, \mu) \in \widetilde{W}_{k}^{N}(a, b) \Longleftrightarrow(\mu, \lambda) \in W_{k}^{N}(b, a) .
$$

Moreover, for any set $W_{k}^{N}(a, b), k \geq 0$, one has

$$
\begin{aligned}
(\lambda, \mu) \in W_{k}^{N}(a, b) & \Longleftrightarrow(-\lambda, \mu) \in W_{k}^{N}(-a, b), \\
(\lambda, \mu) \in W_{k}^{N}(a, b) & \Longleftrightarrow(-\lambda,-\mu) \in W_{k}^{N}(-a,-b), \\
(\lambda, \mu) \in W_{k}^{N}(a, b) & \Longleftrightarrow(\lambda,-\mu) \in W_{k}^{N}(a,-b) .
\end{aligned}
$$


By the relations (4.1)-(4.3), we need only to consider $W_{0}^{N}(a, b), \widetilde{W}_{0}^{N}(a, b)$ and

$$
\begin{array}{ll}
\Gamma_{k}^{N}(a, b):=W_{k}^{D}(a, b) \cap\left(\mathbb{R}_{+} \times \mathbb{R}_{+}\right), & k \geq 1, \\
\widetilde{\Gamma}_{k}^{N}(a, b):=\widetilde{W}_{k}^{D}(a, b) \cap\left(\mathbb{R}_{+} \times \mathbb{R}_{+}\right), & k \geq 1 .
\end{array}
$$

Theorem 4.2 The set $W_{0}^{N}(a, b)$ is made up of two vertical lines $0 \times \mathbb{R}$ and $\lambda_{0}^{N}(a) \times \mathbb{R}$. And $\widetilde{W}_{0}^{N}(a, b)$ is made up of two horizontal lines $\mathbb{R} \times 0$ and $\mathbb{R} \times \lambda_{0}^{N}(b)$. If $\lambda_{0}^{N}(a)$ or $\lambda_{0}^{N}(b)$ does not exist, the corresponding straight line $\lambda_{0}^{N}(a) \times \mathbb{R}$ or $\mathbb{R} \times \lambda_{0}^{N}(b)$ should be understood as an empty set.

Theorem 4.3 If $\Gamma_{k}^{N}(a, b) \neq \emptyset, k \geq 1$, then $\Gamma_{k}^{N}(a, b)$ is a continuous, strictly decreasing, hyperbolic like curve

$$
\begin{aligned}
& g_{k}:\left(\alpha_{k},+\infty\right) \longmapsto\left(\beta_{k},+\infty\right), \\
& \lambda \longmapsto \mu=g_{k}(\lambda),
\end{aligned}
$$

with the horizontal asymptotic line $\mu=\beta_{k}$ and the vertical asymptotic line $\lambda=\alpha_{k}$. Moreover, the lower bound of $\alpha_{k}$ and $\beta_{k}$ can be estimated as follows:

$$
\begin{aligned}
& k=2 n, \quad n \geq 1 \quad \Longrightarrow \quad \alpha_{k} \geq \lambda_{n}^{N}(a), \quad \beta_{k} \geq \lambda_{n}^{D}(b) ; \\
& k=2 n-1, \quad n \geq 1 \quad \Longrightarrow \quad \alpha_{k} \geq \lambda_{n}^{N D}(a), \quad \beta_{k} \geq \lambda_{n-1}^{D N}(b) .
\end{aligned}
$$

Theorem 4.4 Let $k \geq 1$. Then:

(i) $\Gamma_{k}^{N}(a, b) \neq \emptyset$ if and only if $\Lambda_{k}^{N}(a, b)$ exists. And $\Gamma_{k}^{N}(a, b)$ is either an empty set or $a$ hyperbolic like curve emanating from $\left(\Lambda_{k}^{N}(a, b), \Lambda_{k}^{N}(a, b)\right)$.

(ii) $\widetilde{\Gamma}_{k}^{N}(a, b) \neq \emptyset$ if and only if $\Lambda_{k}^{N}(b, a)$ exists. And $\widetilde{\Gamma}_{k}^{N}(a, b)$ is either an empty set or $a$ hyperbolic like curve emanating from $\left(\Lambda_{k}^{N}(b, a), \Lambda_{k}^{N}(b, a)\right)$.

From the relation between $\Lambda_{k}^{N}(a, b)$ and $\Lambda_{k}^{N}(b, a)$ as stated in Property 3.2, we obtain the following spectral structure of $\Pi_{p}^{N}(a, b)$.

Theorem 4.5 One of the following three cases must occur.

(i) $\Gamma_{k}^{N}(a, b)=\emptyset$ and $\widetilde{\Gamma}_{k}^{N}(a, b)=\emptyset$ for any $k \geq 1$.

(ii) $\Gamma_{k}^{N}(a, b) \neq \emptyset$ and $\widetilde{\Gamma}_{k}^{N}(a, b) \neq \emptyset$ for any $k \geq 1$.

(iii) There exist $k_{0} \geq 1$ such that either

$$
\Gamma_{k}^{N}(a, b)\left\{\begin{array} { l l } 
{ \neq \emptyset , } & { 1 \leq k \leq k _ { 0 } , } \\
{ = \emptyset , } & { k > k _ { 0 } , }
\end{array} \widetilde { \Gamma } _ { k } ^ { N } ( a , b ) \left\{\begin{array}{ll}
\neq \emptyset, & 1 \leq k \leq k_{0}-1 \\
=\emptyset, & k \geq k_{0}
\end{array}\right.\right.
$$

or

$$
\Gamma_{k}^{N}(a, b)\left\{\begin{array} { l l } 
{ \neq \emptyset , } & { 1 \leq k \leq k _ { 0 } - 1 , } \\
{ = \emptyset , } & { k \geq k _ { 0 } , }
\end{array} \quad \widetilde { \Gamma } _ { k } ^ { N } ( a , b ) \left\{\begin{array}{ll}
\neq \emptyset, & 1 \leq k \leq k_{0} \\
=\emptyset, & k>k_{0} .
\end{array}\right.\right.
$$

From the relations (4.1)-(4.3) and the above theorems, the structure of the Neumann Fučík spectrum $\Pi_{p}^{N}(a, b)$ becomes clear. 
Theorem 4.6 Let $a, b \in L^{1}[0,1]$. Then $\Pi_{p}^{N}(a, b)$ is composed of (at most) four trivial lines $0 \times \mathbb{R}, \Lambda_{0}^{N}(a) \times \mathbb{R}, \mathbb{R} \times 0, \mathbb{R} \times \Lambda_{0}^{N}(b)$ (if one of the involved principal eigenvalues does not exist, the corresponding straight line is understood as an empty set), and in each quadrant of $\mathbb{R}^{2}$ zero, a finite odd number of, or a double sequence of hyperbolic like curves.

Finally, if the weights $a$ and $b$ are positive, then neither $\lambda_{0}^{N}(a)$ nor $\lambda_{0}^{N}(b)$ exists, and we have the following results.

Theorem 4.7 Let $a, b \in L^{1}[0,1], a>0$ and $b>0$. Then $\Pi_{p}^{N}(a, b)$ is made up of one vertical line, one horizontal line and a double sequence of differentiable, strictly decreasing, hyperbolic like curves in the quadrant $\mathbb{R}^{+} \times \mathbb{R}^{+}$:

$$
\Pi_{p}^{D}(a, b)=(0 \times \mathbb{R}) \cup(\mathbb{R} \times 0) \bigcup_{k \geq 1}\left(\Gamma_{k}^{N}(a, b) \cup \widetilde{\Gamma}_{k}^{N}(a, b)\right) .
$$

If $k=2 n, n \geq 1$, the hyperbolic like curve $\Gamma_{k}^{N}(a, b)$ has the asymptotic lines

$$
\lambda=\lambda_{n}^{N}(a), \quad \mu=\lambda_{n}^{D}(b) .
$$

If $k=2 n-1, n \geq 1$, the hyperbolic like curve $\Gamma_{k}^{N}(a, b)$ has the asymptotic lines

$$
\lambda=\lambda_{n}^{N D}(a), \quad \mu=\lambda_{n-1}^{D N}(b) .
$$

\section{Competing interests}

The authors declare that they have no competing interests.

\section{Authors' contributions}

PY gave the idea of this article and drafted the manuscript. All authors discussed the methods of proving the main results. All authors read and approved the final manuscript.

\section{Author details}

'Department of Mathematics, College of Science, Hohai University, Nanjing, 210098, People's Republic of China.

${ }^{2}$ Department of Mathematical Sciences, Tsinghua University, Beijing, 100084, People's Republic of China.

\section{Acknowledgements}

Jifeng Chu was supported by the National Natural Science Foundation of China (Grant No. 11171090, No. 11271078 and No. 11271333), China Postdoctoral Science Foundation funded project (Grant No. 2012T50431) and the Alexander von Humboldt Foundation of Germany. Ping Yan was supported by the National Natural Science Foundation of China (Grant No. 10901089, No. 11171090 and No. 11371213). Meirong Zhang was supported by the National Natural Science Foundation of China (Grant No. 1123001) and the National 111 Project of China (Station No. 111-2-01).

Received: 18 November 2013 Accepted: 11 December 2013 Published: 09 Jan 2014

\section{References}

1. Dancer, N: On the Dirichlet problem for weakly nonlinear elliptic differential equations. Proc. R. Soc. Edinb. 76, 283-300 (1977)

2. Fučík, S: Boundary value problems with jumping nonlinearities. Čas. Pěst. Mat. 101, 69-87 (1976)

3. Perera, K: On the Fučík spectrum of the $p$-Laplacian. Nonlinear Differ. Equ. Appl. 11, 259-270 (2004)

4. Chen, W, Chu, J, Yan, P, Zhang, M: Complete structure of the Fučík spectrum of the $p$-Laplacian with integrable potentials on an interval. Preprint

5. Alif, M: Sur le spectre de Fučik du p-Laplacien avec des poids indéfinis. C. R. Math. Acad. Sci. Paris 334, 1061-1066 (2002)

6. Lindqvist, P: Some remarkable sine and cosine functions. Ric. Mat. XLIV, 269-290 (1995)

7. Zhang, M: The rotation number approach to eigenvalues of the one-dimensional $p$-Laplacian with periodic potentials. J. Lond. Math. Soc. (2) 64, 125-143 (2001)

8. Li, W, Yan, P: Continuity and continuous differentiability of half-eigenvalues in potentials. Commun. Contemp. Math. $12,977-996(2010)$ 
9. Meng, $G$, Yan, $\mathrm{P}$, Zhang, M: Spectrum of one-dimensional $p$-Laplacian with an indefinite integrable weight. Mediterr. J. Math. 7, 225-248 (2010)

10. Li, W, Yan, P: Various half-eigenvalues of scalar $p$-Laplacian with indefinite integrable weights. Abstr. Appl. Anal. (2009). doi:10.1155/2009/109757

10.1186/1687-2770-2014-10

Cite this article as: Chen et al.: On the Fučík spectrum of the scalar $p$-Laplacian with indefinite integrable weights. Boundary Value Problems 2014, 2014:10

Submit your manuscript to a SpringerOpen ${ }^{\circ}$ journal and benefit from:

- Convenient online submission

- Rigorous peer review

- Immediate publication on acceptance

- Open access: articles freely available online

- High visibility within the field

- Retaining the copyright to your article

Submit your next manuscript at $\boldsymbol{s p r i n g e r o p e n . c o m ~}$ 